ESAIM: M2AN 46 (2012) 841-874

DOI: $10.1051 / \mathrm{m} 2 \mathrm{an} / 2011071$
ESAIM: Mathematical Modelling and Numerical Analysis

www.esaim-m2an.org

\title{
THEORY AND NUMERICAL APPROXIMATIONS FOR A NONLINEAR $1+1$ DIRAC SYSTEM
}

\author{
Nikolaos Bournaveas ${ }^{1}$ And Georgios E. Zouraris ${ }^{2}$
}

\begin{abstract}
We consider a nonlinear Dirac system in one space dimension with periodic boundary conditions. First, we discuss questions on the existence and uniqueness of the solution. Then, we propose an implicit-explicit finite difference method for its approximation, proving optimal order a priori error estimates in various discrete norms and showing results from numerical experiments.
\end{abstract}

Mathematics Subject Classification. 35L40, 35L50, 35Q41, 65M06, 65M12, 65M15, 81Q05.

Received March 18, 2011.

Published online February 3, 2012.

\section{INTRODUCTION}

\subsection{Statement of the problem}

In the work at hand we shall consider a nonlinear Dirac system of equations formulated as follows:

$$
\begin{gathered}
u_{t}+w_{x}=i \alpha_{1} u+i \lambda_{1} f(u, w) u, \\
w_{t}+u_{x}=i \alpha_{2} w+i \lambda_{2} f(u, w) w,
\end{gathered}
$$

where $u=u(x, t)$ and $w=w(x, t)$ are functions of $x \in \mathbb{R}$ and $t \geq 0$, which, for $t=0$, are periodic in $x$ with period $L$. The constants $\alpha_{1}, \alpha_{2}, \lambda_{1}, \lambda_{2}$ are real and $f$ is a smooth real-valued function. This system is a generalization of a physical model for extended particles (see [2]) where $\alpha_{2}=-\alpha_{1}=m \geq 0$ is the mass, $\lambda_{1}=-\lambda_{2}=\lambda$ is a coupling constant and $f(u, w)=|u|^{2}-|w|^{2}$. Then (1.1a)-(1.1b) becomes

$$
\begin{gathered}
u_{t}+w_{x}=-i m u+i \lambda\left(|u|^{2}-|w|^{2}\right) u, \\
w_{t}+u_{x}=i m w-i \lambda\left(|u|^{2}-|w|^{2}\right) w .
\end{gathered}
$$

Here, we have made two basic choices. The first one was to consider periodic initial conditions which was motivated from the observation that several authors developed, tested and analyzed numerical methods for that case (see for example $[7,8,13,25]$ ). The second one was to work with a general nonlinearity since we would develop and analyze a numerical method for the approximation of the solution to the problem which will not be limited by a special type of nonlinearity.

Keywords and phrases. Existence, uniqueness, finite difference methods, error estimates.

1 Department of Mathematics, University of Edinburgh, JCMB, King's Buildings, Edinburgh, EH9 3JZ, Scotland, UK. N.Bournaveas@ed.ac.uk

2 Department of Mathematics, University of Crete, 71409 Heraklion, Crete, Greece. zouraris@math.uoc.gr 
Let us introduce some notation that will be useful in writting (1.1) in a form that is customary in the study of the Dirac equation. We define the Dirac matrices $\gamma^{0}$ and $\gamma^{1}$ by

$$
\gamma^{0}=\left(\begin{array}{ll}
0 & 1 \\
1 & 0
\end{array}\right) \quad \text { and } \quad \gamma^{1}=\left(\begin{array}{cc}
i & 0 \\
0 & -i
\end{array}\right) \text {. }
$$

Then

$$
\gamma^{\mu} \gamma^{\nu}+\gamma^{\nu} \gamma^{\mu}=2 g^{\mu \nu} I_{2 \times 2} \quad \forall \mu, \nu \in\{0,1\}
$$

and

$$
\left(\gamma^{0}\right)^{*}=\gamma^{0}, \quad\left(\gamma^{1}\right)^{*}=-\gamma^{1},
$$

where $\left(g^{\mu \nu}\right)$ is the Minkowski metric, $g^{00}=1, g^{11}=-1$ and $g^{01}=g^{10}=0$. We may of course choose as Dirac matrices any other pair $\gamma^{0}, \gamma^{1}$ which satisfy (1.3) and (1.4). All such pairs are unitarily equivalent.

Let $\psi(x, t)=\left(\psi_{1}(x, t), \psi_{2}(x, t)\right)^{T} \in \mathbb{C} \times \mathbb{C}$ and $m \geq 0$. The linear Dirac equation in one space dimension is the following equation

$$
-i\left(\gamma^{0} \partial_{t} \psi+\gamma^{1} \partial_{x} \psi\right)+m \psi=0
$$

and analogously the nonlinear Dirac equation in one space dimension is formulated as

$$
-i\left(\gamma^{0} \partial_{t} \psi+\gamma^{1} \partial_{x} \psi\right)+m \psi=f(\psi)
$$

If we define

$$
\alpha=-\gamma^{0} \gamma^{1}=\left(\begin{array}{rr}
0 & i \\
-i & 0
\end{array}\right), \quad \beta=-i \gamma^{0}=\left(\begin{array}{rr}
0 & -i \\
-i & 0
\end{array}\right)
$$

then

$$
\alpha^{2}=I, \quad \beta^{2}=-I, \quad \alpha \beta+\beta \alpha=0, \quad \alpha^{*}=\alpha, \quad \beta^{*}=-\beta
$$

and we can write the Dirac equation (1.5) as

$$
\partial_{t} \psi-\alpha \partial_{x} \psi-m \beta \psi=0 .
$$

Now we wish to write the system (1.1) in a similar form. To this end we define

$$
\psi_{1}=u, \quad \psi_{2}=i w .
$$

Then, it is easily seen that (1.1) is equivalent to

$$
\partial_{t} \psi-\alpha \partial_{x} \psi=i A \psi+i f(\psi) \Lambda \psi,
$$

where

$$
A=\left(\begin{array}{cc}
\alpha_{1} & 0 \\
0 & \alpha_{2}
\end{array}\right), \quad \Lambda=\left(\begin{array}{cc}
\lambda_{1} & 0 \\
0 & \lambda_{2}
\end{array}\right)
$$

The Dirac equation arises in relativistic quantum mechanics and describes spin- $1 / 2$ particles, for example electrons. It can be thought of as a relativistic analogue of the Schrödinger equation. Coupled systems such as the Dirac-Klein-Gordon equations and the Maxwell-Dirac equations, as well as nonlinear Dirac equations of the form (1.6), play a fundamental role in physics. We refer the reader to [26] for a detailed discussion of these issues.

The nonlinear Dirac equation (1.6) has been studied both with 'general' nonlinearities $f(\psi)$ and with nonlinearities with special structure [4-6,17-21]. In one space dimension Delgado [9] studied the Cauchy problem for the Thirring model and the Federbusch model (a $4 \times 4$ system of two coupled nonlinear Dirac equations), as well as the Dirac-Klein-Gordon and the Maxwell-Dirac equations, and proves global existence of $\mathrm{H}^{1}$ solutions. 
Glassey [11] studied two $4 \times 4$ systems each consisting of two coupled Dirac equations and proved global existence in $\mathrm{H}^{1}$ under a smallness condition on the initial data.

The plan of the paper is as follows. We will first prove local existence for (1.1) and then investigate whether the local solution can be extended to a global one. We shall show that if $\lambda_{1}=\lambda_{2}$ we have global existence without the need for any smallness assumptions. This is due to a cancellation property of the nonlinearity, similar to the one used in Deldado [9]. In the case $\lambda_{1}=-\lambda_{2}$ a smallness condition in $\mathrm{L}^{2}$ is needed for global existence, in the same spirit as Glassey [11]. We shall then propose a second order, unconditionally stable implicit-explicit finite difference method to construct numerical approximations of the solution to the Dirac system (1.1) for which we prove convergence in the discrete $\mathrm{L}^{\infty}$ norm. Also, we shall discuss the implementation of the proposed method and show results from numerical experiments.

\subsection{Existence theorems}

We begin by defining the notions of solution we shall use.

Definition 1.1. A local $\mathrm{H}^{\ell}$-solution of (1.8) is a 2-spinor field $\psi:(-\infty,+\infty) \times[0, T] \rightarrow \mathbb{C} \times \mathbb{C}, L$-periodic in $x$, with $\psi \in \mathrm{C}^{0}\left([0, T] ; \mathrm{H}_{p e r}^{\ell} \times \mathrm{H}_{p e r}^{\ell}\right)$ which satisfies (1.8) in the sense of distributions.

Definition 1.2. A global $\mathrm{H}^{\ell}$-solution of (1.8) is a 2-spinor field $\psi:(-\infty,+\infty) \times[0, \infty) \rightarrow \mathbb{C} \times \mathbb{C}, L$-periodic in $x$, with $\psi \in \mathrm{C}^{0}\left([0, \infty) ; \mathrm{H}_{\text {per }}^{\ell} \times \mathrm{H}_{\text {per }}^{\ell}\right)$ which satisfies (1.8) in the sense of distributions.

We shall always assume that $\ell$ is a positive integer. Of course if $\ell \geq 2$ an $\mathrm{H}^{\ell}$-solution is automatically continuously differentiable and hence it is a classical solution.

We shall prove the following existence theorems:

Theorem 1.3 (local existence). Let $\psi_{0} \in \mathrm{H}_{\text {per }}^{\ell} \times \mathrm{H}_{\text {per }}^{\ell}$ be a given L-periodic 2-spinor. Then there exists a $T>0$, such that the system $(1.8)$ has a unique $\mathrm{H}^{\ell}$-solution in $(-\infty,+\infty) \times[0, T]$ with $\psi(x, 0)=\psi_{0}(x)$.

Theorem 1.4 (global existence). If $\lambda_{1}=\lambda_{2}$, then the solution of Theorem 1.3 is actually global.

Theorem 1.5 (global existence). Suppose $\alpha_{1}=-\alpha_{2}, \lambda_{1}=-\lambda_{2}$ and $f(u, w)=|u|^{2}-|w|^{2}$. If in addition the smallness condition

$$
\left|\lambda_{1}\right| \int_{0}^{L}\left|\psi_{0}(x)\right|^{2} \mathrm{~d} x<\frac{1}{4}
$$

is satisfied, then the solution of Theorem 1.3 is global.

It is a remarkable fact that only the $\mathrm{L}^{2}$-norm of the initial data enters the smallness condition in Theorem 1.5.

\subsection{An implicit-explicit finite difference method}

The numerical approximation of the solution to the Dirac system (1.1) or (1.2) has been addressed by several authors. In particular, Alvarez and Carreras [2] consider the physical problem (1.2) and investigate, via numerical computations, the interaction dynamics for solitary waves. Alvarez et al. [3] formulate the numerical method used in [2], which combines a second order finite diffence discretization in space with a Crank-Nicolson time stepping, and provide a local-time error estimate in the discrete $\mathrm{L}^{2}$ norm. A complete error analysis in the discrete $\mathrm{L}^{2}$ norm for the Crank-Nicolson finite difference method and in the case of periodic boundary conditions is given by De Frutos [7], who also proves the discrete $\mathrm{L}^{2}$ convergence of an explicit leap-frog finite difference method for (1.2) with periodic boundary conditions and of an implicit box-method for (1.2) with a special type of boundary conditions. De Frutos and Sanz-Serna [8] prove $\mathrm{L}^{2}$ convergence of a split-step spectral method for the Dirac system (1.1) with periodic boundary conditions, $-\lambda_{1}=\lambda_{2}=1$ and a nonlinearity of the form $f(u, w)=\widetilde{f}\left(|u|^{2}-|w|^{2}\right)$. Also, for problem (1.2), Alvarez [1] proposes a linearization of the Crank-Nicolson finite difference method and Jiménez [15] formulates two implicit conservative finite difference methods. Guo et al. [12] 
consider spectral and pseudospectral semidiscrete approximations of the solution to the Cauchy problem for (1.2), proving a local-time discrete $\mathrm{L}^{2}$ error estimate ( $c f$. [3]). Shao and Tang [25] discretize (1.2) using a discontinuous finite element method in space and an explicit Runge-Kutta method in time. Also, they show results from numerical experiments adopting periodic boundary conditions. Hong and Li [13] introduce multisymplectic Runge-Kutta methods for the discretization of (1.2) under periodic boundary conditions, and then discuss their conservation properties and how well a discrete conservation law approximates the corresponding continuous one. Finally, Wang and Tang [27] discretize (1.2) using an explicit second order Runge-Kutta method in time along with a second order finite volume method in space, and propose an adaptive mesh redistribution algorithm. In their numerical experiments they adopt non-reflecting boundary conditions at artificial boundaries. Closing the presentation of the existing bibliography, we would like to point out that the works $[1,13,15,25,27]$ do not provide a mathematical proof for the convergence of the numerical methods proposed, in addition to the computational evidence for their efficiency.

In the work at hand we consider the Dirac system (1.1) under periodic boundary conditions. For the approximation of its solution we propose a numerical method which is different from other methods in the bibliography and combines a second order central finite difference space-discretization with a second order, two-step, implicit-explicit time-stepping method of Crank-Nicolson-type. The term 'implicit-explicit' reflects the fact that the adopted time-stepping method treats the linear part of the system implicitly and the nonlinear one explicitly. The motivation to apply that discretization splitting was the observation that the corresponding linear problem (i.e. the case $f=0$ ) is $\mathrm{L}^{2}$-conservative. Thus, discretizing it with a conservative implicit method (since explicit methods do not have in general conservative properties) we obtain an unconditionally invertible linear discrete operator. Combining it with an explicit discretization of the nonlinear part of the system, the method becomes well-defined without mesh conditions, because only the discrete linear part of the system has to be inverted at every time-step. Thus, we avoid on the one hand CFL conditions required when using explicit methods ( $c f .[7,25,27])$ and on the other hand the iterations needed to solve nonlinear systems of algebraic equations which is the outcome of an implicit method ( $c f .[3,7,8,13,15])$.

Let us formulate our method. First, choose $N \in \mathbb{N}$ and $J \in \mathbb{N}$. Then, introduce a uniform partition of the time interval $[0, T]$ with mesh-length $\tau:=\frac{T}{N}$ and nodes $\mathcal{K}=\left(t_{m}\right)_{m=0}^{N}$ defined by $t_{m}:=m \tau$ for $m=0, \ldots, N$. Also, introduce a uniform partition of the space interval $[0, L]$ with mesh-length $h:=\frac{L}{J+1}$ and nodes $\mathcal{H}=\left(x_{j}\right)_{j=0}^{J+1}$, defined by $x_{j}:=j h$ for $j=0, \ldots, J+1$. The partition $\mathcal{H}$ extends to the whole real line by $x_{j+m(J+1)}:=m L+x_{j}$ for $j=0, \ldots, J$ and $m \in \mathbb{Z}$. In what follows, the finite dimensional space

$$
X_{h}:=\left\{\left(z_{m}\right)_{m \in \mathbb{Z}} \subset \mathbb{C}: \quad z_{m}=z_{m+J+1} \quad \forall m \in \mathbb{Z}\right\},
$$

consisting of periodic complex sequences with period $J+1$, will be the space of the finite difference approximations. For $n=0, \ldots, N$, define the sequences $u^{n}, w^{n} \in X_{h}$, by $u_{j}^{n}:=u\left(x_{j}, t_{n}\right)$ and $w_{j}^{n}:=w\left(x_{j}, t_{n}\right)$ for $j \in \mathbb{Z}$, where the functions $u$ and $w$ form the $L$-periodic solution pair of the continuous problem (1.1a)(1.1b). The implicit-explicit finite difference method we propose constructs, for $m=0, \ldots, N$, an approximation $\left(U^{m}, W^{m}\right) \in X_{h} \times X_{h}$ of $\left(u^{m}, w^{m}\right)$, following the steps below:

Step 1. Set

$$
U_{j}^{0}:=u_{j}^{0} \quad \text { and } \quad W_{j}^{0}:=w_{j}^{0}, \quad j=1, \ldots, J+1 .
$$

Step 2. Find $\left(U^{1}, W^{1}\right) \in X_{h}$ such that

$$
\begin{aligned}
& \frac{U_{j}^{1}-U_{j}^{0}}{\tau}+\frac{1}{2}\left(\frac{W_{j+1}^{1}-W_{j-1}^{1}}{2 h}+\frac{W_{j+1}^{0}-W_{j-1}^{0}}{2 h}\right)=i \alpha_{1} \frac{U_{j}^{1}+U_{j}^{0}}{2}+i \lambda_{1} f\left(U_{j}^{0}, W_{j}^{0}\right) U_{j}^{0}, \\
& \frac{W_{j}^{1}-W_{j}^{0}}{\tau}+\frac{1}{2}\left(\frac{U_{j+1}^{1}-U_{j-1}^{1}}{2 h}+\frac{U_{j+1}^{0}-U_{j-1}^{0}}{2 h}\right)=i \alpha_{2} \frac{W_{j}^{1}+W_{j}^{0}}{2}+i \lambda_{2} f\left(U_{j}^{0}, W_{j}^{0}\right) W_{j}^{0}
\end{aligned}
$$

for $j=1, \ldots, J+1$. 
Step 3. For $n=2, \ldots, N$, find $\left(U^{n}, W^{n}\right) \in X_{h}$ such that

$$
\begin{aligned}
& \frac{U_{j}^{n}-U_{j}^{n-2}}{2 \tau}+\frac{1}{2}\left(\frac{W_{j+1}^{n}-W_{j-1}^{n}}{2 h}+\frac{W_{j+1}^{n-2}-W_{j-1}^{n-2}}{2 h}\right)=i \alpha_{1} \frac{U_{j}^{n}+U_{j}^{n-2}}{2}+i \lambda_{1} f\left(U_{j}^{n-1}, W_{j}^{n-1}\right) U_{j}^{n-1}, \\
& \frac{W_{j}^{n}-W_{j}^{n-2}}{2 \tau}+\frac{1}{2}\left(\frac{U_{j+1}^{n}-U_{j-1}^{n}}{2 h}+\frac{U_{j+1}^{n-2}-U_{j-1}^{n-2}}{2 h}\right)=i \alpha_{2} \frac{W_{j}^{n}+W_{j}^{n-2}}{2}+i \lambda_{2} f\left(U_{j}^{n-1}, W_{j}^{n-1}\right) W_{j}^{n-1}
\end{aligned}
$$

for $j=1, \ldots, J+1$.

The above finite difference method is well-defined with no restrictions on $\tau$ and $h$ (see Sect. 3.2). Also, at every time step the implementation of the method results the need to solve 3-diagonal linear systems of algebraic equations with dimension $\frac{J+1}{2}$ provided that $J+1$ is an even integer (see Sect. 4.1). In the latter situation, the method becomes semi-explicit in the sense that we are able to compute half of the unknowns implicitly by solving linear systems of equations and then to compute the other half one explicitly via formulas that connect them to the previously computed values (see (4.1)). Another characteristic of the method is that, for $n \geq 2$, the matrix of the resulting linear systems is the same at every iteration (see Sect. 4.1) in contrast to the linearized Crank-Nicolson method proposed in [1]. This is achieved since: (i) the partition of the time interval $[0, T]$ is uniform, (ii) the coefficients of the linear part of the equations are time-independent and (iii) the nonlinear part of the equations does not contribute in the matrix of the linear system which is the goal of the implicit-explicit construction of the method.

Investigating the convergence of the finite difference method (1.9)-(1.11), we prove an optimal order error estimate in the discrete $\mathrm{L}^{\infty}(0, L)$-norm (see Thm. 3.13), i.e., that there exists a nonnegative constant $C$ being independent of $\tau$ and $h$, and such that

$$
\max _{0 \leq n \leq N}\left(\max _{1 \leq j \leq J+1}\left|U_{j}^{n}-u_{j}^{n}\right|+\max _{1 \leq j \leq J+1}\left|W_{j}^{n}-w_{j}^{n}\right|\right) \leq C\left(\tau^{2}+h^{2}\right),
$$

provided that $\tau$ and $h$ are small enough. The technique used is based on the construction of a $\delta$-modified finite difference method (see Sect. 3.4) which follows from the finite difference method (1.9)-(1.11) modifying properly the nonlinear terms $(c f$. [28]). The $\delta$-modified finite difference method has two characteristics: (i) its nonlinearity is of Lipshitz-type and (ii) when their approximations are bounded by $\delta$ then it concides to the finite difference method (1.9)-(1.11). First, we derive an optimal order error estimate for the $\delta$-modified finite difference approximations in the discrete $\mathrm{L}^{2}(0, L)$-norm and in the discrete $\mathrm{H}^{1}(0, L)$-seminorm (see Props. 3.8 and 3.12). Then, using a discrete Sobolev inequality (see Lem. 3.1) and assuming that $\tau$ and $h$ are small enough, we are able to keep the discrete $\mathrm{L}^{\infty}(0, L)$-norm of the $\delta$-modified finite difference approximations less than $\delta$, for $\delta$ greater than a mesh-independent value. Thus, the $\delta$-modified finite difference approximations coincide to the approximations of the original finite difference method (1.9)-(1.11) (see Lem. 3.3), and the error estimates for the modified finite difference method hold also for the original finite difference method. We would like to stress that our analysis avoids the usual mesh condition $\tau=o\left(h^{\frac{1}{4}}\right)(c f$. Rem. 3.11) used in other works on the analysis of numerical methods for problem (1.2) or (1.1) in order to show convergence in a discrete $\mathrm{L}^{2}$ norm (see, e.g., $[7,8]$ ). Also, the error analysis presented in $[3,12]$ assumes that the final time $T$ is small enough. Finally, in Section 4, we explain implementation issues for our finite difference method, and we show results from numerical experiments that confirm the order of convergence of the method.

In the work at hand, we propose and investigate an implicit-explicit finite difference method for problem (1.1) with second order accuracy in space and time. Higher-order numerical methods of implicit-explicit-type could be formulated by combining a properly chosen Runge-Kutta or multistep method for time-discretization, with a finite element or a finite volume method for space-discretization. The development and the analysis of such methods could be the object of a future research, taking into account that since the Dirac system has an hyperbolic character the order of convergence of a higher order method may faces optimality limitations (see, e.g., $[10])$. 


\section{Proofs of the EXISTEnCE THEOREMS}

\subsection{Linear estimates}

We shall base the proof of the local existence theorem for (1.8) on the following estimates for the linear system (1.7) with $m=0$. These estimates are well known in the 'non-periodic' case and their proofs in the periodic case are very similar. We shall therefore be brief.

Proposition 2.1 (conservation of charge). Let $\psi$ be a L-periodic $\mathrm{H}^{1}$-solution of

$$
\partial_{t} \psi-\alpha \partial_{x} \psi=0 \text {. }
$$

Then for all $t$, it holds that

$$
\int_{0}^{L}|\psi(x, t)|^{2} \mathrm{~d} x=\int_{0}^{L}|\psi(x, 0)|^{2} \mathrm{~d} x .
$$

Proof. Multiply the equation by $\psi^{\dagger}$, the conjugate transpose of $\psi$, and take the real part of the resulting equation to get $\partial_{t}\left(\psi^{\dagger} \psi\right)-\partial_{x}\left(\psi^{\dagger} \alpha \psi\right)=0$. Integrate over $[0, L]$ and use the periodicity condition to get $\partial_{t} \int_{0}^{L} \psi^{\dagger} \psi \mathrm{d} x=0$. This implies (2.1).

Conservation of charge implies the following two estimates. Their role in the theory of the Dirac equation is similar to the role of the energy estimates in the theory of the wave equation.

Proposition 2.2 (charge estimate). Let $\psi$ be a L-periodic, $\mathrm{H}^{1}$-solution of the non-homogeneous system

$$
\partial_{t} \psi-\alpha \partial_{x} \psi=G
$$

with initial data $\psi(x, 0)=\psi_{0}(x)$. Then, for all $t \geq 0$, it holds that

$$
\|\psi(\cdot, t)\|_{\mathrm{L}^{2}(0, L)} \leq\left\|\psi_{0}\right\|_{\mathrm{L}^{2}(0, L)}+\int_{0}^{t}\|G(\cdot, \tau)\|_{\mathrm{L}^{2}(0, L)} \mathrm{d} \tau
$$

Proof. In the special case $G=0$ the result follows immediately from (2.1). In the special case $\psi_{0}=0$ we can use Duhamel's principle to get $\psi(x, t)=\int_{0}^{t} \phi(x, t-s ; s) \mathrm{d} s$, where $\phi(x, t ; s)$ is defined as the unique solution of $\partial_{t} \phi(x, t ; s)-\alpha \partial_{x} \phi(x, t ; s)=0, \phi(x, 0 ; s)=G(x, s)$. Then, we have

$$
\begin{aligned}
\|\psi(t, \cdot)\|_{\mathrm{L}^{2}(0, L)} & \leq \int_{0}^{t}\|\phi(\cdot, t-s ; s)\|_{\mathrm{L}^{2}(0, L)} \mathrm{d} s \\
& =\int_{0}^{t}\|\phi(\cdot, 0 ; s)\|_{\mathrm{L}^{2}(0, L)} \mathrm{d} s \\
& =\int_{0}^{t}\|G(\cdot, s)\|_{\mathrm{L}^{2}(0, L)} \mathrm{d} s .
\end{aligned}
$$

The result in the general case follows easily from these two special cases.

Proposition 2.3 (generalized charge estimate). Let $\psi$ be a L-periodic, $\mathrm{H}^{\ell}$-solution of the non-homogeneous system

$$
\partial_{t} \psi-\alpha \partial_{x} \psi=G
$$

with initial data $\psi(x, 0)=\psi_{0}(x)$. Then for all $t \geq 0$,

$$
\|\psi(\cdot, t)\|_{\mathrm{H}^{\ell}(0, L)} \leq\left\|\psi_{0}\right\|_{\mathrm{H}^{\ell}(0, L)}+\int_{0}^{t}\|G(\cdot, \tau)\|_{\mathrm{H}^{\ell}(0, L)} \mathrm{d} \tau .
$$


Proof. Differentiate the equation and apply (2.2).

We shall also need the following Moser-type Calculus Inequalities.

Lemma 2.4. The following inequalities hold:

(1) if $f, g \in \mathrm{H}_{\text {per }}^{\ell} \cap \mathrm{L}^{\infty}$ then

$$
\|f g\|_{\mathrm{H}^{\ell}(0, L)} \leq C\left(\|f\|_{\mathrm{H}^{\ell}(0, L)}\|g\|_{\mathrm{L}^{\infty}}+\|g\|_{\mathrm{H}^{\ell}(0, L)}\|f\|_{\mathrm{L}^{\infty}}\right)
$$

(2) if $F$ is smooth on a domain $G, u$ is continuous with $u(x) \in \overline{G_{1}} \subset \subset G$ and $u \in \mathrm{H}_{\text {per }}^{\ell} \cap \mathrm{L}^{\infty}$ then

$$
\|F \circ u\|_{\mathrm{H}^{\ell}(0, L)} \leq C\left(\sum_{|\alpha| \leq \ell}\left\|\partial^{\alpha} F\right\|_{L^{\infty}\left(\overline{G_{1}}\right)}\right)\|u\|_{\mathrm{L}^{\infty}}^{\ell-1}\|u\|_{\mathrm{H}^{\ell}(0, L)} .
$$

Proof. [22], page 43 and [14], page 108 discuss the case of $\mathrm{H}^{\ell}\left(\mathbb{R}^{n}\right)$. The proofs in that case can easily be adapted to the periodic setting.

Of course, if $n=1$ and $\ell \geq 1$ the above $\mathrm{L}^{\infty}$-norms can be estimated by the corresponding $\mathrm{H}^{\ell}$-norms.

\subsection{Local existence for the nonlinear system}

Existence results for Dirac systems are usually proven using the abstract methods of [16,24], see for example [23]. Here we shall use a more direct method based on the generalized charge estimate. This line of proof has been used in the theory of nonlinear wave equations where the main tools are Generalized Energy Estimates.

Proof of Theorem 1.3. Throughout this proof the letter $C$ will always denote either an absolute constant or a constant which may depend on $A, \Lambda$ or $f$ but is otherwise independent of the initial data $\psi_{0}$ and may change from line to line.

Let $\psi_{0} \in \mathrm{H}_{p e r}^{\ell} \times \mathrm{H}_{p e r}^{\ell}$ be given. Fix $T>0$. Smallness conditions on $T$ will be imposed in the course of the proof. Define

$$
X=\left\{\psi \in \mathrm{C}^{0}\left([0, T] ; \mathrm{H}_{p e r}^{\ell} \times \mathrm{H}_{p e r}^{\ell}\right): \sup _{0 \leq t \leq T}\|\psi(\cdot, t)\|_{\mathrm{H}^{\ell}(0, L)} \leq 2\left\|\psi_{0}\right\|_{\mathrm{H}^{\ell}(0, L)}\right\} .
$$

Then $X$ is a ball in the Banach space $\mathrm{C}^{0}\left([0, T] ; \mathrm{H}_{\text {per }}^{\ell} \times \mathrm{H}_{\text {per }}^{\ell}\right)$ with the norm

$$
\|\psi\|_{X}=\sup _{0 \leq t \leq T}\|\psi(\cdot, t)\|_{\mathrm{H}^{\ell}(0, L)} .
$$

We define an operator $\mathcal{T}: X \rightarrow X$ as follows: let $\psi \in X$. Since

$$
\int_{0}^{T}\|(i A \psi+i \Lambda f(\psi) \psi)(\cdot, \tau)\|_{\mathrm{H}^{\ell}(0, L)} \mathrm{d} \tau<+\infty
$$

the theory of the linear Dirac equation guarantees that the initial value problem

$$
\begin{gathered}
\partial_{t} z-\alpha \partial_{x} z=i A \psi+i \Lambda f(\psi) \psi, \\
z(x, 0)=\psi_{0}(x)
\end{gathered}
$$

has a unique solution $z \in \mathrm{C}^{0}\left([0, T], \mathrm{H}_{\text {per }}^{\ell} \times \mathrm{H}_{\text {per }}^{\ell}\right)$. We define $\mathcal{T} \psi:=z$. To make sure that that $\mathcal{T} \psi \in X$ we need to show

$$
\sup _{0 \leq t \leq T}\|z(\cdot, t)\|_{\mathrm{H}^{\ell}(0, L)} \leq 2\left\|\psi_{0}\right\|_{\mathrm{H}^{\ell}(0, L)} .
$$


To prove this we use the generalized charge estimate (2.3), as follows:

$$
\begin{aligned}
\|z(\cdot, t)\|_{\mathrm{H}^{\ell}(0, L)} & \leq\left\|\psi_{0}\right\|_{\mathrm{H}^{\ell}(0, L)}+\int_{0}^{T}\|(i A \psi+i \Lambda f(\psi) \psi)(\cdot, \tau)\|_{\mathrm{H}^{\ell}(0, L)} \mathrm{d} \tau \\
& \leq\left\|\psi_{0}\right\|_{\mathrm{H}^{\ell}(0, L)}+C T\|\psi\|_{X}+C \int_{0}^{T}\|(f(\psi) \psi)(\cdot, \tau)\|_{\mathrm{H}^{\ell}(0, L)} \mathrm{d} \tau \\
& \leq\left\|\psi_{0}\right\|_{\mathrm{H}^{\ell}(0, L)}(1+2 C T)+C \int_{0}^{T}\|(f(\psi) \psi)(\cdot, \tau)\|_{\mathrm{H}^{\ell}(0, L)} \mathrm{d} \tau .
\end{aligned}
$$

We need to estimate the last term in (2.7). We have:

$$
\begin{aligned}
\|f(\psi(\cdot, \tau)) \psi(\cdot, \tau)\|_{\mathrm{H}^{\ell}(0, L)} \leq & C\|f(\psi(\cdot, \tau))\|_{\mathrm{H}^{\ell}(0, L)}\|\psi(\cdot, \tau)\|_{\mathrm{L}^{\infty}(0, L)} \\
& +C\|f(\psi(\cdot, \tau))\|_{\mathrm{L}^{\infty}(0, L)}\|\psi(\cdot, \tau)\|_{\mathrm{H}^{\ell}(0, L)} .
\end{aligned}
$$

Since we are working in one space dimension and we are assuming that $\ell \geq 1$, the $\mathrm{L}^{\infty}$ norm is controlled by the $\mathrm{H}^{\ell}$ norm. Therefore

$$
\|f(\psi(\cdot, \tau)) \psi(\cdot, \tau)\|_{\mathrm{H}^{\ell}(0, L)} \leq C\|f(\psi(\cdot, \tau))\|_{\mathrm{H}^{\ell}(0, L)}\|\psi(\cdot, \tau)\|_{\mathrm{H}^{\ell}(0, L)} .
$$

Now $\psi \in X$ therefore

$$
\|\psi(\cdot, \tau)\|_{\mathrm{H}^{\ell}(0, L)} \leq\|\psi\|_{X} \leq 2\left\|\psi_{0}\right\|_{\mathrm{H}^{\ell}(0, L)} .
$$

Let $B=\left\{w \in \mathbb{C} \times \mathbb{C}:|w| \leq C\left\|\psi_{0}\right\|_{\mathrm{H}^{\ell}(0, L)}\right\}$. Then, by (2.5),

$$
\begin{aligned}
\|f(\psi(\cdot, \tau))\|_{\mathrm{H}^{\ell}(0, L)} & \leq C\left(\sum_{|\alpha| \leq s}\left\|\partial^{\alpha} f\right\|_{\mathrm{L}^{\infty}(B)}\right)\|\psi(\cdot, \tau)\|_{\mathrm{L}^{\infty}(0, L)}^{\ell-1}\|\psi(\cdot, \tau)\|_{\mathrm{H}^{\ell}(0, L)} \\
& \leq C\|\psi(\cdot, \tau)\|_{\mathrm{H}^{\ell}(0, L)}^{\ell} \\
& \leq C\|\psi\|_{X}^{\ell} \\
& \leq C\left\|\psi_{0}\right\|_{\mathrm{H}^{\ell}(0, L)}^{\ell} .
\end{aligned}
$$

Using (2.9) and (2.10) in (2.8), we have

$$
\|f(\psi(\cdot, \tau)) \psi(\cdot, \tau)\|_{\mathrm{H}^{\ell}(0, L)} \leq C\left\|\psi_{0}\right\|_{\mathrm{H}^{\ell}(0, L)}^{\ell+1} .
$$

Therefore for last term of (2.7) we have

$$
\int_{0}^{T}\|(f(\psi) \psi)(\cdot, \tau)\|_{\mathrm{H}^{\ell}(0, L)} \mathrm{d} \tau \leq C T\left\|\psi_{0}\right\|_{\mathrm{H}^{\ell}(0, L)}^{\ell+1} .
$$

Using (2.11) in (2.7) we get

$$
\|z(\cdot, t)\|_{\mathrm{H}^{\ell}(0, L)} \leq\left\|\psi_{0}\right\|_{\mathrm{H}^{\ell}(0, L)}\left(1+2 C T+C T\left\|\psi_{0}\right\|_{\mathrm{H}^{\ell}(0, L)}^{\ell}\right) .
$$

If $T$ is small enough so that

$$
2 C T+C T\left\|\psi_{0}\right\|_{\mathrm{H}_{(0, L)}^{\ell}}^{\ell} \leq 1,
$$

then

$$
\|z(\cdot, t)\|_{\mathrm{H}^{\ell}(0, L)} \leq 2\left\|\psi_{0}\right\|_{\mathrm{H}^{\ell}(0, L)} .
$$

This estimate proves that $\mathcal{T}(\psi)=z \in X$.

Our next aim is to show that $\mathcal{T}$ is a contraction. The proof is actually very similar to what we have already done. We shall use $D_{0}$ to denote any constant that depends on the $\mathrm{H}^{\ell}$ norm of the initial data. It is not essential for our purposes to keep track of the exact dependence of $D_{0}$ on $\left\|\psi_{0}\right\|_{\mathrm{H}^{\ell}(0, L)}$. 
Let $\psi, \zeta \in X$. Then $\mathcal{T}(\psi)-\mathcal{T}(\zeta)$ satisfies

$$
\begin{aligned}
\left(\partial_{t}-\alpha \partial_{x}\right)(\mathcal{T}(\psi)-\mathcal{T}(\zeta)) & =i A(\psi-\zeta)+i(f(\psi)-f(\zeta)) \Lambda \psi+i f(\zeta) \Lambda(\psi-\zeta) \\
(\mathcal{T}(\psi)-\mathcal{T}(\zeta))(x, 0) & =0
\end{aligned}
$$

Using (2.3) we have

$$
\|\mathcal{T}(\psi)-\mathcal{T}(\zeta)\|_{X} \leq \int_{0}^{T}\|i A(\psi-\zeta)+i f(\zeta) \Lambda(\psi-\zeta)+i(f(\psi)-f(\zeta)) \Lambda \psi\|_{\mathrm{H}^{\ell}(0, L)} \mathrm{d} t
$$

For the first term in right hand side we have

$$
\int_{0}^{T}\|i A(\psi(\cdot, t)-\zeta(\cdot, t))\|_{\mathrm{H}^{\ell}(0, L)} \mathrm{d} t \leq C T\|\psi-\zeta\|_{X} .
$$

For the second term we have

$$
\begin{aligned}
\|i f(\zeta(\cdot, t)) \Lambda(\psi(\cdot, t)-\zeta(\cdot, t))\|_{\mathrm{H}^{\ell}(0, L)} \leq & C\|f(\zeta(\cdot, t))\|_{\mathrm{H}^{\ell}(0, L)}\|\psi(\cdot, t)-\zeta(\cdot, t)\|_{\mathrm{L}^{\infty}(0, L)} \\
& +C\|f(\zeta(\cdot, t))\|_{\mathrm{L}^{\infty}(0, L)}\|\psi(\cdot, t)-\zeta(\cdot, t)\|_{\mathrm{H}^{\ell}(0, L)} \\
\leq & C\|f(\zeta(\cdot, t))\|_{\mathrm{H}^{\ell^{(}(0, L)}}\|\psi(\cdot, t)-\zeta(\cdot, t)\|_{\mathrm{H}^{\ell}(0, L)} .
\end{aligned}
$$

Working as above we find

$$
\|f(\zeta(\cdot, t))\|_{\mathrm{H}^{\ell}(0, L)} \leq C D_{0},
$$

therefore

$$
\|i f(\zeta(\cdot, t)) \Lambda(\psi(\cdot, t)-\zeta(\cdot, t))\|_{\mathrm{H}^{\ell}(0, L)} \leq C D_{0}\|\psi-\zeta\|_{X} .
$$

Thus for the second term in the right hand side of (2.12) we have:

$$
\int_{0}^{T}\|i f(\zeta(\cdot, t)) \Lambda(\psi(\cdot, t)-\zeta(\cdot, t))\|_{\mathrm{H}^{\ell}(0, L)} \mathrm{d} t \leq C D_{0} T\|\psi-\zeta\|_{X} .
$$

Working similarly with the third term in the right hand side of (2.12) we get:

$$
\int_{0}^{T}\|i(f(\psi(\cdot, t))-f(\zeta(\cdot, t))) \Lambda \psi(\cdot, t)\|_{\mathrm{H}^{\ell}(0, L)} \leq C D_{0} T\|\psi-\zeta\|_{X} .
$$

Using (2.16), (2.15) and (2.13) in (2.12) we get

$$
\|\mathcal{T} \psi-\mathcal{T} \zeta\|_{X} \leq\left(C+C D_{0}\right) T\|\psi-\zeta\|_{X} .
$$

Therefore, if $T$ is small enough so that $\left(C+C D_{0}\right) T<1$, then $\mathcal{T}$ is a contraction.

This completes the proof of existence in Theorem 1.3. Uniqueness follows from similar arguments.

Remark 2.5. Using similar arguments one can show that

$$
\partial_{t} \psi, \partial_{x} \psi \in \mathrm{C}^{k}\left([0, T] ; \mathrm{H}_{\text {per }}^{\ell-k} \times \mathrm{H}_{\text {per }}^{\ell-k}\right), \quad 0 \leq k \leq \ell .
$$

\subsection{Global existence for the nonlinear system}

\subsubsection{Proof of Theorem 1.4}

It is well known that the global existence claim of Theorem 1.4 follows from the a-priori estimate of the following proposition. We only deal with the case $\ell=1$ because higher values of $\ell$ can be treated by differentiating the equation and proving similar estimates in a standard way. The proof uses an observation of Delgado [9]. 
Proposition 2.6. Suppose $\lambda_{1}=\lambda_{2}$. Let $\psi \in \mathrm{C}^{0}\left([0, T) ; \mathrm{H}_{p e r}^{1} \times \mathrm{H}_{\text {per }}^{1}\right)$ be a solution of (1.8) in some time interval $[0, T)$ with $T<\infty$. Then

$$
\sup _{0 \leq t<T}\|\psi(\cdot, t)\|_{\mathrm{H}^{1}(0, L)}<\infty .
$$

Proof. The plan of the proof is as follows: we first obtain an $\mathrm{L}^{\infty}$ estimate using integration along characteristics. We then use this $\mathrm{L}^{\infty}$ estimate together with 'charge estimates' to prove an $\mathrm{H}^{1}$ estimate. In both cases a Gronwall argument is used. The hypothesis $\lambda_{1}=\lambda_{2}$ is crucial as it results in a cancellation thanks to which the proof works without any smallness assumptions. We shall use the letter $C$ for all constants which may depend on $A$, $\Lambda, T, L$ or $f$ but are independent of $\psi_{0}$, and the symbol $D_{0}$ for constants which depend on $\left\|\psi_{0}\right\|_{\mathrm{H}^{1}(0, L)}$.

Multiply (1.8) by $\psi^{\dagger}$, the conjugate transpose of $\psi$, and take the real part of the resulting equation to get

$$
\partial_{t} J^{0}+\partial_{x} J^{1}=0
$$

where

$$
J^{0}=\psi^{\dagger} \psi, \quad J^{1}=-\psi^{\dagger} \alpha \psi
$$

Equation (2.18) expresses conservation of charge. Now multiply (1.8) by $\psi^{\dagger} \alpha$ and take the real part of the resulting equation to get

$$
\partial_{t} J^{1}+\partial_{x} J^{0}=i \psi^{\dagger}\left((\alpha A)^{*}-\alpha A\right) \psi+i f(\psi) \psi^{\dagger}\left((\alpha \Lambda)^{*}-\alpha \Lambda\right) \psi .
$$

We have $(\alpha \Lambda)^{*}-\alpha \Lambda=\left(\lambda_{1}-\lambda_{2}\right)\left(\begin{array}{ll}0 & i \\ i & 0\end{array}\right)=0$ therefore

$$
\partial_{t} J^{1}+\partial_{x} J^{0}=i \psi^{\dagger}\left((\alpha A)^{*}-\alpha A\right) \psi .
$$

The system consisting of (2.18) and (2.19) can easily be integrated along characteristics to give

$$
\begin{aligned}
J^{0}(x, t)= & \frac{1}{2}\left[J_{0}^{0}(x-t)+J_{0}^{1}(x-t)+J_{0}^{0}(x+t)-J_{0}^{1}(x+t)\right] \\
& +i \int_{0}^{t}[k(x-t+s, s)-k(x+t-s, s)] \mathrm{d} s,
\end{aligned}
$$

where

$$
J_{0}^{\mu}(x)=J^{\mu}(x, 0), \quad \mu=0,1,
$$

and

$$
k=\psi^{\dagger}\left((\alpha A)^{*}-\alpha A\right) \psi
$$

We have

$$
\begin{gathered}
J^{0}=|\psi|^{2}, \\
\left|J_{0}^{\mu}(x)\right| \leq C\left\|\psi_{0}\right\|_{\mathrm{L}_{(0, L)}^{\infty}}^{2} \leq C\left\|\psi_{0}\right\|_{\mathrm{H}^{1}(0, L)}^{2}
\end{gathered}
$$

and

$$
|k(x, t)| \leq C\|\psi(\cdot, t)\|_{\mathrm{L}^{\infty}(0, L)}^{2} .
$$

Therefore, taking the $\mathrm{L}^{\infty}$ norm in $(2.20)$ we get

$$
\|\psi(\cdot, t)\|_{\mathrm{L}^{\infty}(0, L)}^{2} \leq C\left\|\psi_{0}\right\|_{\mathrm{H}^{1}(0, L)}^{2}+C \int_{0}^{t}\|\psi(\cdot, \tau)\|_{\mathrm{L}^{\infty}(0, L)}^{2} \mathrm{~d} \tau
$$


and Gronwall's lemma gives

$$
\begin{aligned}
\|\psi(\cdot, t)\|_{\mathrm{L}^{\infty}(0, L)} & \leq C \mathrm{e}^{C t}\left\|\psi_{0}\right\|_{\mathrm{H}^{1}(0, L)} \\
& \leq C \mathrm{e}^{C T}\left\|\psi_{0}\right\|_{\mathrm{H}^{1}(0, L)} \\
& \leq C D_{0} .
\end{aligned}
$$

This is an a-priori estimate for the $\mathrm{L}^{\infty}$ norm of $\psi$. Combining it with the generalized charge estimate we shall now prove the desired a-priori estimate for the $\mathrm{H}^{1}$ norm. Indeed, applying (2.3) to (1.8) we get

$$
\begin{aligned}
\|\psi(\cdot, t)\|_{\mathrm{H}^{1}(0, L)} \leq & \left\|\psi_{0}\right\|_{\mathrm{H}^{1}(0, L)}+\int_{0}^{t}\|(i A \psi+i \Lambda f(\psi) \psi)(\cdot, \tau)\|_{\mathrm{H}^{1}(0, L)} \mathrm{d} \tau \\
\leq & \left\|\psi_{0}\right\|_{\mathrm{H}^{1}(0, L)}+C \int_{0}^{t}\|\psi(\cdot, \tau)\|_{\mathrm{H}^{1}(0, L)} \mathrm{d} \tau \\
& +C \int_{0}^{t}\|f(\psi(\cdot, \tau)) \psi(\cdot, \tau)\|_{\mathrm{H}^{1}(0, L)} \mathrm{d} \tau
\end{aligned}
$$

For the last term in $(2.22)$ we have:

$$
\|f(\psi(\cdot, \tau)) \psi(\cdot, \tau)\|_{\mathrm{H}^{1}(0, L)} \leq\|f(\psi(\cdot, \tau)) \psi(\cdot, \tau)\|_{\mathrm{L}^{2}(0, L)}+\left\|\partial_{x}(f(\psi(\cdot, \tau)) \psi(\cdot, \tau))\right\|_{\mathrm{L}^{2}(0, L)} .
$$

Let $B$ be the ball in $\mathbb{C} \times \mathbb{C}$ centered at the origin and of radius equal to the constant $C D_{0}$ in the last line of estimate (2.21). Then

$$
\begin{aligned}
\|f(\psi(\cdot, \tau)) \psi(\cdot, \tau)\|_{\mathrm{L}^{2}(0, L)} & \leq C\|f(\psi(\cdot, \tau))\|_{\mathrm{L}^{\infty}(0, L)}\|\psi(\cdot, \tau)\|_{\mathrm{L}^{2}(0, L)} \\
& \leq C\|f\|_{\mathrm{L}^{\infty}(B)}\|\psi(\cdot, \tau)\|_{\mathrm{L}^{2}(0, L)} \\
& \leq C\|\psi(\cdot, \tau)\|_{\mathrm{H}^{1}(0, L)} .
\end{aligned}
$$

(The constant $C$ depends on the initial data through the radius of the ball $B$ ). On the other hand

$$
\left\|\partial_{x}(f(\psi(\cdot, \tau)) \psi(\cdot, \tau))\right\|_{\mathrm{L}^{2}(0, L)} \leq\left\|\partial_{x}(f(\psi(\cdot, \tau))) \psi(\cdot, \tau)\right\|_{\mathrm{L}^{2}(0, L)}+\left\|f(\psi(\cdot, \tau)) \partial_{x} \psi(\cdot, \tau)\right\|_{\mathrm{L}^{2}(0, L)} .
$$

For the first term in the right hand side of $(2.25)$ we have

$$
\begin{aligned}
\left\|\partial_{x}(f(\psi(\cdot, \tau))) \psi(\cdot, \tau)\right\|_{\mathrm{L}^{2}(0, L)} & \leq\left\|\partial_{x}(f(\psi(\cdot, \tau)))\right\|_{\mathrm{L}^{2}(0, L)}\|\psi(\cdot, \tau)\|_{\mathrm{L}^{\infty}(0, L)} \\
& \leq C\left[\sum_{|\alpha| \leq 1}\left\|\partial^{\alpha} f\right\|_{\mathrm{L}^{\infty}(B)}\right]\left\|\partial_{x} \psi(\cdot, \tau)\right\|_{\mathrm{L}^{2}(0, L)}\|\psi(\cdot, \tau)\|_{\mathrm{L}^{\infty}(0, L)} \\
& \leq C D_{0}\|\psi(\cdot, \tau)\|_{\mathrm{H}^{1}(0, L)} .
\end{aligned}
$$

For the second term in the righthand side of $(2.25)$ we have

$$
\begin{aligned}
\left\|f(\psi(\cdot, \tau)) \partial_{x} \psi(\cdot, t)\right\|_{\mathrm{L}^{2}(0, L)} & \leq\|f(\psi(\cdot, \tau))\|_{\mathrm{L}^{\infty}(0, L)}\left\|\partial_{x} \psi(\cdot, \tau)\right\|_{\mathrm{L}^{2}(0, L)} \\
& \leq C\|\psi(\cdot, \tau)\|_{\mathrm{H}^{1}(0, L)} .
\end{aligned}
$$

(The constant $C$ depends on the initial data through the radius of the ball $B$ ). Using (2.27) and (2.26) in (2.25) we have

$$
\left\|\partial_{x}(f(\psi(\cdot, \tau)) \psi(\cdot, \tau))\right\|_{\mathrm{L}^{2}(0, L)} \leq C D_{0}\|\psi(\cdot, \tau)\|_{\mathrm{H}^{1}(0, L)} .
$$

Using (2.28) and (2.24) in (2.23) we get

$$
\|f(\psi(\cdot, \tau)) \psi(\cdot, \tau)\|_{\mathrm{H}^{1}(0, L)} \leq C D_{0}\|\psi(\cdot, \tau)\|_{\mathrm{H}^{1}(0, L)} .
$$


Therefore for the last term in $(2.22)$ we have

$$
\int_{0}^{t}\|f(\psi(\cdot, \tau)) \psi(\cdot, \tau)\|_{\mathrm{H}^{1}(0, L)} \mathrm{d} \tau \leq C D_{0} \int_{0}^{t}\|\psi(\cdot, \tau)\|_{\mathrm{H}^{1}(0, L)} \mathrm{d} \tau .
$$

Using (2.30) in (2.22) we finally get that, for all $t \in[0, T]$,

$$
\|\psi(\cdot, t)\|_{\mathrm{H}^{1}(0, L)} \leq\left\|\psi_{0}\right\|_{\mathrm{H}^{1}(0, L)}+C D_{0} \int_{0}^{t}\|\psi(\cdot, \tau)\|_{\mathrm{H}^{1}(0, L)} \mathrm{d} \tau
$$

and Grönwall's Lemma gives, for all $t \in[0, T]$,

$$
\|\psi(\cdot, t)\|_{\mathrm{H}^{1}(0, L)} \leq C D_{0} \mathrm{e}^{C D_{0} t}
$$

with constants depending on $T$. This completes the proof.

\subsubsection{Proof of Theorem 1.5}

To prove our second global existence theorem we use a technique of Glassey [11]. It suffices to handle the case $\ell=1$ since higher $\ell$ can be treated by differentiating the equation and proving similar estimates. It is well known that it is enough to prove the a-priori estimate contained in the following proposition.

Proposition 2.7. Suppose $\alpha_{1}=-\alpha_{2}, \lambda_{1}=-\lambda_{2}, f(u, w)=|u|^{2}-|w|^{2}$ and

$$
\left|\lambda_{1}\right| \int_{0}^{L}\left|\psi_{0}(x)\right|^{2} \mathrm{~d} x<\frac{1}{4}
$$

Let $\psi \in \mathrm{C}^{0}\left([0, T) ; \mathrm{H}_{\text {per }}^{1} \times \mathrm{H}_{\text {per }}^{1}\right)$ be a solution of $(1.8)$ in some time interval $[0, T)$ with $T<\infty$. Then

$$
\sup _{0 \leq t<T}\|\psi(\cdot, t)\|_{\mathrm{H}^{1}(0, L)}<\infty .
$$

Proof. Since we need to diagonalize our system we might as well work directly with (1.1) instead of (1.8). Define a new 2 -spinor field $\zeta$ by

$$
u=\zeta_{1}+\zeta_{2}, \quad w=\zeta_{1}-\zeta_{2} .
$$

Set

$$
\lambda_{1}=-\lambda_{2}=\lambda, \quad \alpha_{1}=-\alpha_{2}=-m .
$$

Then (1.1) becomes

$$
\begin{aligned}
& \partial_{t} \zeta_{1}+\partial_{x} \zeta_{1}=-i m \zeta_{2}+4 i \lambda \operatorname{Re}\left(\zeta_{1} \overline{\zeta_{2}}\right) \zeta_{2}, \\
& \partial_{t} \zeta_{2}-\partial_{x} \zeta_{2}=-i m \zeta_{1}+4 i \lambda \operatorname{Re}\left(\zeta_{1} \overline{\zeta_{2}}\right) \zeta_{1}
\end{aligned}
$$

In matrix form we can write this system as

$$
\partial_{t} \zeta=B \partial_{x} \zeta-i m \gamma^{0} \zeta+4 i g(\zeta) \gamma^{0} \zeta
$$

where

$$
B=\left(\begin{array}{rr}
-1 & 0 \\
0 & 1
\end{array}\right) \quad, \quad \gamma^{0}=\left(\begin{array}{ll}
0 & 1 \\
1 & 0
\end{array}\right), \quad g(\zeta)=\operatorname{Re}\left(\zeta_{1} \overline{\zeta_{2}}\right) .
$$

The continuity equation for the conservation of charge now takes the form

$$
\partial_{t}\left(\left|\zeta_{1}\right|^{2}+\left|\zeta_{2}\right|^{2}\right)-\partial_{x}\left(-\left|\zeta_{1}\right|^{2}+\left|\zeta_{2}\right|^{2}\right)=0
$$


From this we get the law of conservation of charge

$$
\forall t \in[0, T): \quad \int_{0}^{L}|\zeta(x, t)|^{2} \mathrm{~d} x=\int_{0}^{L}\left|\zeta_{0}(x)\right|^{2} \mathrm{~d} x
$$

where $\zeta_{0}(x)=\zeta(x, 0)$ is the initial data of $\zeta$.

Fix $(x, t) \in \mathbb{R} \times[0, T)$. Following [11] we integrate (2.38) over the backward 'cone' $C(x, t)$ with tip at $(x, t)$ and 'base' on the $x$-axis,

$$
C(x, t)=\left\{\left(x^{\prime}, t^{\prime}\right): 0 \leq t^{\prime} \leq t,\left|x-x^{\prime}\right| \leq t-t^{\prime}\right\}
$$

(this is simply a triangle in one space dimension) and use Green's theorem to get:

$$
2 \int_{0}^{t}\left(\left|\zeta_{1}(x+t-s, s)\right|^{2}+\left|\zeta_{2}(x-t+s, s)\right|^{2}\right) \mathrm{d} s=\int_{x-t}^{x+t}\left|\zeta_{0}(y)\right|^{2} \mathrm{~d} y .
$$

Suppose now that $T \leq L$, in other words, suppose that the strip $\mathbb{R} \times[0, T)$ has height $\leq L$. Then the interval $[x-t, x+t]$ in the right hand side of $(2.40)$ has length $\leq 2 L$. We have $[x-t, x+t] \subseteq[x-L, x+L]$ therefore the following 'cone estimate' is true:

$$
\begin{aligned}
2 \int_{0}^{t}\left(\left|\zeta_{1}(x+t-s, s)\right|^{2}+\left|\zeta_{2}(x-t+s, s)\right|^{2}\right) \mathrm{d} s & \leq \int_{x-L}^{x+L}\left|\zeta_{0}(y)\right|^{2} \mathrm{~d} y \\
& =\int_{0}^{2 L}\left|\zeta_{0}(y)\right|^{2} \mathrm{~d} y=2 \int_{0}^{L}\left|\zeta_{0}(y)\right|^{2} \mathrm{~d} y
\end{aligned}
$$

Now fix an arbitrary point $\left(x_{0}, t_{0}\right) \in \mathbb{R} \times[0, T)$ and consider the backward 'cone' $C_{0}$ with tip $\left(x_{0}, t_{0}\right)$ and 'base' on the $x$-axis,

$$
C_{0}=C\left(x_{0}, t_{0}\right)=\left\{(x, t): 0 \leq t \leq t_{0},\left|x-x_{0}\right| \leq t_{0}-t\right\} .
$$

We wish to estimate $\sup _{C_{0}}\left|\zeta_{1}\right|$. This quantity is finite because $C_{0}$ is compact and $\zeta$ is continuous. Integrating along characteristics we find that for any $(x, t) \in C_{0}$,

$$
\begin{aligned}
\zeta_{1}(x, t)= & \zeta_{1}(x-t, 0)-i m \int_{0}^{t} \zeta_{2}(x-t+s, s) \mathrm{d} s \\
& +4 i \lambda \int_{0}^{t} \operatorname{Re}\left(\zeta_{1}(x-t+s, s) \overline{\zeta_{2}(x-t+s, s)}\right) \zeta_{2}(x-t+s, s) \mathrm{d} s .
\end{aligned}
$$

Therefore

$$
\begin{aligned}
\left|\zeta_{1}(x, t)\right| \leq & \left\|\zeta_{10}\right\|_{L^{\infty}(\mathbb{R})}+m \sqrt{t}\left(\int_{0}^{t}\left|\zeta_{2}(x-t+s, s)\right|^{2} \mathrm{~d} s\right)^{\frac{1}{2}} \\
& +4|\lambda| \int_{0}^{t}\left|\zeta_{1}(x-t+s, s)\right|\left|\zeta_{2}(x-t+s, s)\right|^{2} \mathrm{~d} s \\
\leq & \left\|\zeta_{10}\right\|_{L^{\infty}(\mathbb{R})}+m \sqrt{t}\left(\int_{0}^{t}\left|\zeta_{2}(x-t+s, s)\right|^{2} \mathrm{~d} s\right)^{\frac{1}{2}} \\
& +4|\lambda|\left(\int_{0}^{t}\left|\zeta_{2}(x-t+s, s)\right|^{2} \mathrm{~d} s\right) \sup _{C_{0}}\left|\zeta_{1}\right|,
\end{aligned}
$$

where $\zeta_{10}(x)=\zeta_{1}(x, 0)$. Using the cone estimate $(2.41)$ we get

$$
\left|\zeta_{1}(x, t)\right| \leq\left\|\zeta_{10}\right\|_{L^{\infty}(\mathbb{R})}+m \sqrt{t}\left\|\zeta_{0}\right\|_{L^{2}(0, L)}+4|\lambda|\left\|\zeta_{0}\right\|_{L^{2}(0, L)}^{2} \sup _{C_{0}}\left|\zeta_{1}\right|
$$


and since $(x, t)$ was an arbitrary point in $C_{0}$, we have shown:

$$
\sup _{C_{0}}\left|\zeta_{1}\right| \leq\left\|\zeta_{10}\right\|_{L^{\infty}(\mathbb{R})}+m \sqrt{t}\left\|\zeta_{0}\right\|_{L^{2}(0, L)}+4|\lambda|\left\|\zeta_{0}\right\|_{L^{2}(0, L)}^{2} \sup _{C_{0}}\left|\zeta_{1}\right| .
$$

The smallness condition (2.32) implies

$$
4|\lambda|\left\|\zeta_{0}\right\|_{\mathrm{L}^{2}(0, L)}^{2}<\frac{1}{2}
$$

therefore

$$
\sup _{C_{0}}\left|\zeta_{1}\right| \leq 2\left\|\zeta_{10}\right\|_{L^{\infty}(\mathbb{R})}+2 m \sqrt{t}\left\|\zeta_{0}\right\|_{L^{2}(0, L)} .
$$

Since $C_{0}$ was an arbitrary 'cone' in $\mathbb{R} \times[0, T)$ we conclude

$$
\sup _{\mathbb{R} \times[0, T)}\left|\zeta_{1}\right| \leq 2\left\|\zeta_{10}\right\|_{L^{\infty}(\mathbb{R})}+2 m \sqrt{T}\left\|\zeta_{0}\right\|_{L^{2}(0, L)}
$$

and therefore

$$
\sup _{\mathbb{R} \times[0, T)}\left|\zeta_{1}\right|<\infty
$$

This is an a-priori $\mathrm{L}^{\infty}$ estimate for $\zeta_{1}$ which was proven under the assumption $T \leq L$.

Suppose now that $T>L$. Since $\zeta \in \mathrm{C}^{0}\left([0, T) ; \mathrm{H}_{\text {per }}^{1}\right)$ we have

$$
\sup _{0 \leq t \leq T-L}\|\zeta(\cdot, t)\|_{\mathrm{H}^{1}(0, L)}<\infty .
$$

Sobolev's inequality then implies

$$
\sup _{\mathbb{R} \times[0, T-L]}|\zeta|<\infty .
$$

It remains to estimate the $\mathrm{L}^{\infty}$ norm of $\zeta$ in $\mathbb{R} \times[T-L, T)$. This however is a strip with height $L$ and 'base' at $t=T-L$, and we can repeat the argument we used above to get the following analogue of (2.43):

$$
\sup _{\mathbb{R} \times[T, T-L)}\left|\zeta_{1}\right| \leq 2 \sup _{x \in \mathbb{R}}\left|\zeta_{1}(x, T-L)\right|+2 m \sqrt{T}\|\zeta(\cdot, T-L)\|_{\mathrm{L}^{2}(0, L)}
$$

provided that the following smallness condition is satisfied:

$$
4|\lambda|\|\zeta(\cdot, T-L)\|_{\mathrm{L}^{2}(0, L)}^{2}<\frac{1}{2} .
$$

Thanks to conservation of charge (2.39)

$$
\|\zeta(\cdot, T-L)\|_{\mathrm{L}^{2}(0, L)}^{2}=\left\|\zeta_{0}\right\|_{\mathrm{L}^{2}(0, L)}^{2}
$$

and therefore the left hand side of (2.45) is exactly the same as the left hand side of (2.42). Therefore (2.45) is indeed satisfied. Then (2.44), Sobolev's inequality and (2.46) give

$$
\begin{aligned}
\sup _{\mathbb{R} \times[T, T-L)}\left|\zeta_{1}\right| & \leq 2\left\|\zeta_{1}(\cdot, T-L)\right\|_{\mathrm{H}^{1}(0, L)}+2 m \sqrt{T}\left\|\zeta_{0}\right\|_{\mathrm{L}^{2}(0, L)} \\
& <\infty
\end{aligned}
$$

This completes the proof of the a-priori $\mathrm{L}^{\infty}$ estimate for $\zeta_{1}$. Working similarly we can prove an a-priori $\mathrm{L}^{\infty}$ estimate for $\zeta_{2}$ and thus we have an a-priori $\mathrm{L}^{\infty}$ estimate for the 2 -spinor field $\zeta$, as required. 


\section{Convergence analysis of the finite Difference method}

\subsection{Notation and preliminaries}

In this section, we introduce notation to shorten the mathematical formulas, and present some basic relations often used later.

First, we introduce a set of discrete operators which is described below:

- space-discrete operators: we define a discrete space-derivative operator $\delta_{h}: X_{h} \rightarrow X_{h}$ by

$$
\forall v \in X_{h}: \quad \delta_{h} v_{j}:=\frac{v_{j+1}-v_{j-1}}{2 h}, \quad j=1, \ldots, J+1,
$$

the shift operators $\theta_{h}^{+}, \theta_{h}^{-}: X_{h} \rightarrow X_{h}$ by

$$
\forall v \in X_{h}: \quad \theta_{h}^{+} v_{j}:=v_{j+1} \text { and } \theta_{h}^{-} v_{j}:=v_{j-1}, \quad j=1, \ldots, J+1,
$$

and the product operator $\cdot \otimes \cdot: X_{h} \times X_{h} \rightarrow X_{h}$ by

$$
\forall \omega, v \in X_{h}: \quad(\omega \otimes v)_{j}:=\omega_{j} v_{j}, \quad j=1, \ldots, J+1 ;
$$

- time-discrete operators: for given $\left(S^{m}\right)_{m=1}^{2}$ and $\left(V^{m}\right)_{m=0}^{N} \subset X_{h}$, we define the discrete time-derivative operators $\partial_{\tau}^{\text {init }}$ and $\partial_{\tau}$ and the discrete time-average operators $\mathcal{A}^{\text {init }}$ and $\mathcal{A}$, by

and

$$
\partial_{\tau}^{\text {init }} S^{1}:=\frac{S^{1}-S^{0}}{\tau}, \quad \mathcal{A}^{\text {init }} S^{1}:=\frac{S^{1}+S^{0}}{2}
$$

$$
\partial_{\tau} V^{m}:=\frac{V^{m}-V^{m-2}}{2 \tau}, \quad \mathcal{A} V^{m}:=\frac{V^{m}+V^{m-2}}{2}, \quad m=2, \ldots, N .
$$

Next, we introduce the following notation conventions and simplifications:

- for $\ell \in \mathbb{N}$, any function $g: \mathbb{C}^{\ell} \rightarrow \mathbb{C}$ and any $y=\left(y^{1}, \ldots, y^{\ell}\right) \in\left(X_{h}\right)^{\ell}$ we define $g(y) \in X_{h}$ by

$$
g(y)_{j}:=g\left(y_{j}^{1}, \ldots, y_{j}^{\ell}\right), \quad j=1, \ldots, J+1 ;
$$

- the function $f: \mathbb{C} \times \mathbb{C} \rightarrow \mathbb{R}$ appears in the formulation (1.1) of the continuous problem, will be considered as a function $f_{\mathbb{R}}: \mathbb{R}^{4} \rightarrow \mathbb{R}$, i.e.,

$$
\forall z_{1}, z_{2} \in \mathbb{C}: \quad f\left(z_{1}, z_{2}\right):=f_{\mathbb{R}}\left(\operatorname{Re}\left(z_{1}\right), \operatorname{Im}\left(z_{1}\right), \operatorname{Re}\left(z_{2}\right), \operatorname{Im}\left(z_{2}\right)\right) ;
$$

- for $n=0, \ldots, N$, we define $\left(u^{n}, w^{n}\right)_{n=0}^{N} \subset X_{h} \times X_{h}$ (cf. Sect. 1.3) by

$$
u_{j}^{n}:=u\left(x_{j}, t_{n}\right) \quad \text { and } \quad w_{j}^{n}:=w\left(x_{j}, t_{n}\right), \quad j=1, \ldots, J+1 ;
$$

where the functions $u$ and $w$ form the $L$-periodic solution pair of the continuous problem (1.1a)-(1.1b).

- for any $\varepsilon>0$ we set $K_{\varepsilon}:=[-\varepsilon, \varepsilon] \subset \mathbb{R}$.

Finally, we introduce some norms and an inner product:

- for $\ell \in \mathbb{N}$, we shall consider the following standard norms in $\mathbb{R}^{\ell}:\|x\|_{\infty, \mathbb{R}^{\ell}}:=\max _{1 \leq i \leq \ell}\left|x_{i}\right|,\|x\|_{1, \mathbb{R}^{\ell}}:=$ $\sum_{i=1}^{\ell}\left|x_{i}\right|$ and $\|x\|_{2, \mathbb{R}^{\ell}}:=\left(\sum_{i=1}^{\ell}\left|x_{i}\right|^{2}\right)^{\frac{1}{2}}$ for $x \in \mathbb{R}^{\ell}$

- the space $X_{h}$ is provided with a discrete $\mathrm{L}^{2}(0, L)$-inner product $(\cdot, \cdot)_{0, h}$ defined by

$$
\forall \omega, v \in X_{h}: \quad(\omega, v)_{0, h}:=h \sum_{j=1}^{J+1} \omega_{j} \overline{v_{j}},
$$

inducing a discrete $\mathrm{L}^{2}(0, L)$-norm $\|\cdot\|_{0, h}$ given by $\|v\|_{0, h}:=\sqrt{(v, v)_{0, h}}$ for $v \in X_{h}$. Also, we define a discrete $\mathrm{H}^{1}(0, L)$-seminorm by $|v|_{1, h}:=\left\|\delta_{h} v\right\|_{0, h}$ for $v \in X_{h}$, a discrete $\mathrm{H}^{1}(0, L)$-norm by $\|v\|_{1, h}:=\left(\|v\|_{0, h}^{2}+|v|_{1, h}^{2}\right)^{\frac{1}{2}}$ for $v \in X_{h}$, and a discrete maximum norm by $|v|_{\infty}:=\max _{1 \leq j \leq J+1}\left|v_{j}\right|$ for $v \in X_{h}$. 
The discrete space derivative $\delta_{h}$ satisfies a discrete version of the integration by parts:

$$
\forall v, w \in X_{h}: \quad\left(\delta_{h} v, \omega\right)_{0, h}=-\left(v, \delta_{h} \omega\right)_{0, h}
$$

and the following discrete product differentiation:

$$
\forall v, w \in X_{h}: \quad \delta_{h}(v \otimes w)=\delta_{h} v \otimes \theta_{h}^{+} w+\theta_{h}^{-} v \otimes \delta_{h} w .
$$

We close this section by showing that a discrete Sobolev-type inequality holds.

Lemma 3.1. There exists a real constant $C_{S}>0$, independent of $h$, such that

$$
\forall v \in X_{h}: \quad|v|_{\infty}^{2} \leq C_{S}\|v\|_{0, h}\left(\|v\|_{0, h}+|v|_{1, h}\right) .
$$

Proof. Let $v \in X_{h}$. Then there exists $j_{0} \in\{1, \ldots, J+1\}$ such that $|v|_{\infty}=\left|v_{j_{0}}\right|$. Setting $j_{*}:=j_{0}+3(J+1)$, we have $|v|_{\infty}=\left|v_{j_{*}}\right|$ and $x_{j_{*}} \in(3 L, 4 L]$. Now, we consider the auxilliary quantities $\left(\psi_{j}\right)_{j=0}^{j_{*}} \subset \mathbb{R}$ defined by $\psi_{j}:=\frac{2}{x_{j_{*}}} x_{j}-1$ for $j=0, \ldots, j_{*}$. Thus, we have: (i) $\psi_{0}=-1$, (ii) $\psi_{1}<0$ since $2 x_{1} \leq 2 L<x_{j_{*}}$, (iii) $\psi_{j_{*}}=1$, and (iv) $\psi_{j_{*}-1}>0$ since $2 x_{j_{*}-1} \geq 6 L>x_{j_{*}}$. Also, it is, easily, seen that $\max _{0 \leq j \leq j_{*}}\left|\psi_{j}\right| \leq 1$. Now, we introduce the auxiliary quantity $S_{*}:=\sum_{j=1}^{j_{*}-1}\left(\left|v_{j+1}\right|^{2} \psi_{j+1}-\left|v_{j-1}\right|^{2} \psi_{j-1}\right)$. First we observe that

$$
\begin{aligned}
S_{*} & =\sum_{j=2}^{j_{*}}\left|v_{j}\right|^{2} \psi_{j}-\sum_{j=0}^{j_{*}-2}\left|v_{j}\right|^{2} \psi_{j} \\
& =\left|v_{j_{*}}\right|^{2} \psi_{j_{*}}+\left|v_{j_{*}-1}\right|^{2} \psi_{j_{*}-1}-\left|v_{1}\right|^{2} \psi_{1}-\left|v_{0}\right|^{2} \psi_{0} \\
& \geq\left|v_{j_{*}}\right|^{2}+\left|v_{0}\right|^{2} \\
& \geq|v|_{\infty}^{2} .
\end{aligned}
$$

To derive an upper bound for $S_{*}$ first we observe that

$$
\begin{aligned}
S_{*} & =\sum_{j=1}^{j_{*}-1}\left|v_{j+1}\right|^{2}\left(\psi_{j+1}-\psi_{j-1}\right)+\sum_{j=1}^{j_{*}-1} \psi_{j-1}\left(\left|v_{j+1}\right|^{2}-\left|v_{j-1}\right|^{2}\right) \\
& =\frac{4}{x_{j_{*}}} \sum_{j=1}^{j_{*}-1} h\left|v_{j+1}\right|^{2}+\sum_{j=1}^{j_{*}-1} \psi_{j-1} \operatorname{Re}\left[\left(v_{j+1}-v_{j-1}\right)\left(\overline{v_{j+1}}+\overline{v_{j-1}}\right)\right] .
\end{aligned}
$$

Then, using the properties of $\left(\psi_{j}\right)_{j=0}^{j_{*}}$ and the Cauchy-Schwarz inequality we obtain

$$
\begin{aligned}
S_{*} & \leq \frac{4}{3 L} \sum_{j=1}^{4(J+1)} h\left|v_{j+1}\right|^{2}+2 \sum_{j=1}^{4(J+1)} h\left|\delta_{h} v_{j}\right|\left|v_{j+1}+v_{j-1}\right| \\
& \leq \frac{16}{3 L} \sum_{j=1}^{J+1} h\left|v_{j+1}\right|^{2}+8 \sum_{j=1}^{J+1} h\left|\delta_{h} v_{j}\right|\left|v_{j+1}+v_{j-1}\right| \\
& \leq \frac{16}{3 L}\|v\|_{0, h}^{2}+8 \sqrt{2}\left\|\delta_{h} v\right\|_{0, h}\left(\sum_{j=1}^{J+1} h\left(\left|v_{j+1}\right|^{2}+\left|v_{j-1}\right|^{2}\right)\right)^{\frac{1}{2}}
\end{aligned}
$$

which finally yields

$$
S_{*} \leq \frac{16}{3 L}\|v\|_{0, h}^{2}+16\left\|\delta_{h} v\right\|_{0, h}\|v\|_{0, h} .
$$

Combining (3.5) and (3.6), we arrive at (3.4) with $C_{S}=16 \max \left\{1, \frac{1}{3 L}\right\}$. 


\subsection{The finite difference method is well-defined}

Using the discrete operators introduced in Section 3.1, we formulate the finite difference method described in Section 1.3, as follows:

Step 1. Set

$$
U^{0}:=u^{0} \quad \text { and } \quad W^{0}:=w^{0} .
$$

Step 2. Find $U^{1} \in X_{h}$ and $W^{1} \in X_{h}$ such that

$$
\begin{gathered}
\partial_{\tau}^{\text {init }} U^{1}+\delta_{h} \mathcal{A}^{\text {init }} W^{1}=i \alpha_{1} \mathcal{A}^{\text {init }} U^{1}+i \lambda_{1} f\left(U^{0}, W^{0}\right) \otimes U^{0} \\
\partial_{\tau}^{\text {init }} W^{1}+\delta_{h} \mathcal{A}^{\text {init }} U^{1}=i \alpha_{2} \mathcal{A}^{\text {init }} W^{1}+i \lambda_{2} f\left(U^{0}, W^{0}\right) \otimes W^{0}
\end{gathered}
$$

Step 3. For $n=2, \ldots, N$, find $U^{n} \in X_{h}$ and $W^{n} \in X_{h}$ such that

$$
\begin{gathered}
\partial_{\tau} U^{n}+\delta_{h} \mathcal{A} W^{n}=i \alpha_{1} \mathcal{A} U^{n}+i \lambda_{1} f\left(U^{n-1}, W^{n-1}\right) \otimes U^{n-1}, \\
\partial_{\tau} W^{n}+\delta_{h} \mathcal{A} U^{n}=i \alpha_{2} \mathcal{A} W^{n}+i \lambda_{2} f\left(U^{n-1}, W^{n-1}\right) \otimes W^{n-1} .
\end{gathered}
$$

Now, defining, for $\beta \in \mathbb{R}^{3}$, a linear operator $\Gamma_{h}(\beta ; \cdot): X_{h} \times X_{h} \longrightarrow X_{h} \times X_{h}$ by

$$
\Gamma_{h}\left(\beta ;\left(v_{A}, v_{B}\right)\right):=\left(\left(1-i \tau \beta_{2}\right) v_{A}+\tau \beta_{1} \delta_{h} v_{B},\left(1-i \tau \beta_{3}\right) v_{B}+\tau \beta_{1} \delta_{h} v_{A}\right), \quad \forall\left(v_{A}, v_{B}\right) \in X_{h} \times X_{h},
$$

we, easily, conclude that

$$
\Gamma_{h}\left(\beta_{A} ;\left(U^{1}, W^{1}\right)\right)=\left(i \tau \frac{\alpha_{1}}{2} U^{0}-\frac{\tau}{2} \delta_{h} W^{0}+i \lambda_{1} \tau f\left(U^{0}, W^{0}\right) \otimes U^{0}, i \tau \frac{\alpha_{2}}{2} W^{0}-\frac{\tau}{2} \delta_{h} U^{0}+i \lambda_{2} \tau f\left(U^{0}, W^{0}\right) \otimes W^{0}\right)
$$

and

$$
\begin{aligned}
\Gamma_{h}\left(\beta_{B} ;\left(U^{n}, W^{n}\right)\right)= & \left(i \tau \alpha_{1} U^{n-1}-\tau \delta_{h} W^{n-1}+i 2 \lambda_{1} \tau f\left(U^{n-1}, W^{n-1}\right) \otimes U^{n-1},\right. \\
& \left.i \tau \alpha_{2} W^{n-1}-\tau \delta_{h} U^{n-1}+i 2 \lambda_{2} \tau f\left(U^{n-1}, W^{n-1}\right) \otimes W^{n-1}\right)
\end{aligned}
$$

for $n=2, \ldots, N$, where $\beta_{A}:=\left(\frac{1}{2}, \frac{\alpha_{1}}{2}, \frac{\alpha_{2}}{2}\right)$ and $\beta_{B}:=\left(1, \alpha_{1}, \alpha_{2}\right)$. Thus, the existence and uniqueness of the finite difference approximations follows easily by the invertibility of $\Gamma_{h}(\beta ; \cdot)$ which is the outcome of the following lemma.

Lemma 3.2. For all $\beta \in \mathbb{R}^{3}$, the operator $\Gamma_{h}(\beta ; \cdot)$, defined by $(3.10)$, is invertible.

Proof. Let $\beta \in \mathbb{R}^{3}$. Since the space $X_{h} \times X_{h}$ has finite dimension, the invertibility of the linear operator $\Gamma_{h}(\beta ; \cdot)$ follows by showing that it is one-to-one. For that, let $\left(\omega_{A}, \omega_{B}\right) \in X_{h} \times X_{h}$ such that $\Gamma_{h}\left(\beta ;\left(\omega_{A}, \omega_{B}\right)\right)=0$. Then $\operatorname{Re}\left(\left(1-i \tau \beta_{2}\right) \omega_{A}+\tau \beta_{1} \delta_{h} \omega_{B}, \omega_{A}\right)_{0, h}=0$ and $\operatorname{Re}\left(\left(1-i \tau \beta_{3}\right) \omega_{B}+\tau \beta_{1} \delta_{h} \omega_{A}, \omega_{B}\right)_{0, h}=0$ which yield that

$$
\left\|\omega_{A}\right\|_{0, h}^{2}+\tau \beta_{1} \operatorname{Re}\left(\delta_{h} \omega_{B}, \omega_{A}\right)_{0, h}=0 \quad \text { and } \quad\left\|\omega_{B}\right\|_{0, h}^{2}+\tau \beta_{1} \operatorname{Re}\left(\delta_{h} \omega_{A}, \omega_{B}\right)_{0, h}=0 .
$$

Using (3.2) we conclude that

$$
\operatorname{Re}\left(\delta_{h} \omega_{B}, \omega_{A}\right)_{0, h}=-\operatorname{Re}\left(\delta_{h} \omega_{A}, \omega_{B}\right)_{0, h} .
$$

Thus, (3.11) and (3.12) yield $\left\|\omega_{A}\right\|_{0, h}^{2}+\left\|\omega_{B}\right\|_{0, h}^{2}=0$, or, $\left(\omega_{A}, \omega_{B}\right)=0$, which ends the proof. 


\subsection{Consistency}

Let $\left\{\left(\rho^{n}, \sigma^{n}\right)\right\}_{n=1}^{N} \subset X_{h} \times X_{h}$, be defined by

$$
\begin{aligned}
& \partial_{\tau}^{\text {init }} u^{1}+\delta_{h} \mathcal{A}^{\text {init }} w^{1}=i \alpha_{1} \mathcal{A}^{\text {init }} u^{1}+i \lambda_{1} f\left(u^{0}, w^{0}\right) \otimes u^{0}+\rho^{1}, \\
& \partial_{\tau}^{\text {init }} w^{1}+\delta_{h} \mathcal{A}^{\text {init }} u^{1}=i \alpha_{2} \mathcal{A}^{\text {init }} w^{1}+i \lambda_{2} f\left(u^{0}, w^{0}\right) \otimes w^{0}+\sigma^{1}
\end{aligned}
$$

and

$$
\begin{aligned}
& \partial_{\tau} u^{n}+\delta_{h} \mathcal{A} w^{n}=i \alpha_{1} \mathcal{A} u^{n}+i \lambda_{1} f\left(u^{n-1}, w^{n-1}\right) \otimes u^{n-1}+\rho^{n}, \\
& \partial_{\tau} w^{n}+\delta_{h} \mathcal{A} u^{n}=i \alpha_{2} \mathcal{A} w^{n}+i \lambda_{2} f\left(u^{n-1}, w^{n-1}\right) \otimes w^{n-1}+\sigma^{n}
\end{aligned}
$$

for $n=2, \ldots, N$, where $\left\{\left(u^{n}, w^{m}\right)\right\}_{n=0}^{N} \subset X_{h} \times X_{h}$ have been defined by (3.1). Then, using the Taylor formula, we obtain

$$
\left|\rho^{1}\right|_{\infty}+\left|\sigma^{1}\right|_{\infty} \leq C\left(\tau+h^{2}\right), \quad \max _{2 \leq n \leq N}\left(\left|\rho^{n}\right|_{\infty}+\left|\sigma^{n}\right|_{\infty}\right) \leq C\left(\tau^{2}+h^{2}\right)
$$

and

$$
\left|\delta_{h} \rho^{1}\right|_{\infty}+\left|\delta_{h} \sigma^{1}\right|_{\infty} \leq C\left(\tau+h^{2}\right), \max _{2 \leq n \leq N}\left(\left|\delta_{h} \rho^{n}\right|_{\infty}+\left|\delta_{h} \sigma^{n}\right|_{\infty}\right) \leq C\left(\tau^{2}+h^{2}\right) .
$$

\subsection{A modified finite difference method}

The development of a convergence analysis for the finite difference method (3.7)-(3.9) pass through the efficient handling of the locally-Lipschitz nonlinearity of the problem. Since we are not able to prove for the finite difference approximations an a priori bound in the discrete maximum norm, we introduce and analyze a modified finite difference method which follows from the finite difference method (3.7)-(3.9) after a modification of its nonlinear terms. Our modification is based on the use of a $\delta$-parameter dependent cut-off function $g_{\delta}$, choosen to achieve the following basic effects:

- the nonlinear terms become globally Lipschitz (with a constant that depends on $\delta$ ) (cf. Sect. 3.5) which allows the derivation of error estimates for the modified finite difference approximations, and

- the nonlinear terms remain unaffected for complex numbers belonging to an area around zero with a radius less or equal to $\delta$, and thus it is possible to build-up a condition yielding that the modified finite difference approximations are those of the finite difference method (3.7)-(3.9) (see Lem. 3.3).

In particular, let

$$
\delta_{\star}:=\sup _{[0, T]}\|u\|_{L^{\infty}(0, L)}+\sup _{[0, T]}\|w\|_{L^{\infty}(0, L)}+1
$$

and

$$
\delta>\delta_{\star}
$$

Then, we consider a bounded, monotone increasing function $g_{\delta} \in C^{2}(\mathbb{R} ; \mathbb{R})$ with bounded derivatives up to second order, satisfying

$$
g_{\delta}(x):=\left\{\begin{array} { l l } 
{ x , } & { \text { if } \quad | x | \leq \delta } \\
{ 2 \frac { x } { | x | } \delta , } & { \text { if } \quad | x | > 3 \delta }
\end{array} \text { and } \quad g _ { \delta } ( x ) \in \left\{\begin{array}{lll}
{[\delta, 2 \delta],} & \text { if } \quad x \in[\delta, 3 \delta] \\
{[-2 \delta,-\delta],} & \text { if } \quad x \in[-3 \delta,-\delta]
\end{array}\right.\right.
$$

for $x \in \mathbb{R}$. Next, we define a function $\gamma_{\delta}: \mathbb{C} \rightarrow \mathbb{C}$ by

$$
\gamma_{\delta}(z):=g_{\delta}(\operatorname{Re} z)+i g_{\delta}(\operatorname{Im} z) \quad \forall z \in \mathbb{C},
$$

and the function $f_{\delta}: \mathbb{C} \times \mathbb{C} \rightarrow \mathbb{C}$ by

$$
f_{\delta}\left(z_{1}, z_{2}\right):=f\left(\gamma_{\delta}\left(z_{1}\right), \gamma_{\delta}\left(z_{2}\right)\right), \quad \forall z_{1}, z_{2} \in \mathbb{C} .
$$


It is easily seen that $\left|\gamma_{\delta}(z)\right| \leq 2 \sqrt{2} \delta$ for $z \in \mathbb{C}$ and

$$
\gamma_{\delta}(z)=z \quad \text { for all } z \in \mathbb{C} \text { with }|z| \leq \delta .
$$

The modified finite difference method, for $n=0, \ldots, N$, constructs an approximation $\left(U^{\delta, n}, W^{\delta, n}\right) \in X_{h} \times X_{h}$ of $\left(u^{n}, w^{n}\right)$, following the steps below:

Step 1. Set

$$
U^{\delta, 0}:=u^{0} \quad \text { and } \quad W^{\delta, 0}:=w^{0} .
$$

Step 2. Find $\left(U^{\delta, 1}, W^{\delta, 1}\right) \in X_{h} \times X_{h}$ such that

$$
\begin{gathered}
\partial_{\tau}^{\text {init }} U^{\delta, 1}+\delta_{h} \mathcal{A}^{\text {init }} W^{\delta, 1}=i \alpha_{1} \mathcal{A}^{\text {init }} U^{\delta, 1}+i \lambda_{1} f_{\delta}\left(U^{\delta, 0}, W^{\delta, 0}\right) \otimes \gamma_{\delta}\left(U^{\delta, 0}\right), \\
\partial_{\tau}^{\text {init }} W^{\delta, 1}+\delta_{h} \mathcal{A}^{\text {init }} U^{\delta, 1}=i \alpha_{2} \mathcal{A}^{\text {init }} W^{\delta, 1}+i \lambda_{2} f_{\delta}\left(U^{\delta, 0}, W^{\delta, 0}\right) \otimes \gamma_{\delta}\left(W^{\delta, 0}\right) .
\end{gathered}
$$

Step 3. For $n=2, \ldots, N$, find $\left(U^{\delta, n}, W^{\delta, n}\right) \in X_{h} \times X_{h}$ such that

$$
\begin{gathered}
\partial_{\tau} U^{\delta, n}+\delta_{h} \mathcal{A} W^{\delta, n}=i \alpha_{1} \mathcal{A} U^{\delta, n}+i \lambda_{1} f_{\delta}\left(U^{\delta, n-1}, W^{\delta, n-1}\right) \otimes \gamma_{\delta}\left(U^{\delta, n-1}\right), \\
\partial_{\tau} W^{\delta, n}+\delta_{h} \mathcal{A} U^{\delta, n}=i \alpha_{2} \mathcal{A} W^{\delta, n}+i \lambda_{2} f_{\delta}\left(U^{\delta, n-1}, W^{\delta, n-1}\right) \otimes \gamma_{\delta}\left(W^{\delta, n-1}\right) .
\end{gathered}
$$

The existence and uniqueness of $\left\{\left(U^{\delta, n}, W^{\delta, n}\right)\right\}_{n=1}^{N}$ follows easily by the invertibility of the discrete operator $\Gamma_{h}$ defined in (3.10). Also, (3.17) yields that $\gamma_{\delta}\left(u_{j}^{n}\right)=u_{j}^{n}$ and $\gamma_{\delta}\left(w_{j}^{n}\right)=w_{j}^{n}$ for $n=0, \ldots, N$ and $j \in \mathbb{Z}$. This means that the consistency argument for the modified finite difference method (3.20)-(3.22) is the same to that of the finite difference method (3.7)-(3.9). We close this section by presenting a condition that allows to conclude that the approximations produced by the modified finite difference method (3.20)-(3.22) are those of the finite difference method (3.7)-(3.9).

Lemma 3.3. Let $\delta_{\star}$ be the constant defined by $(3.16), \delta>\delta_{\star},\left\{\left(U^{m}, W^{m}\right)\right\}_{m=0}^{N}$ be the approximations of the finite difference method (3.7)-(3.9) and $\left\{\left(U^{\delta, m}, W^{\delta, m}\right)\right\}_{m=0}^{N}$ be the approximations of the modified finite difference method (3.20)-(3.22). If

$$
\max _{1 \leq n \leq N} \max \left\{\left|U^{\delta, n}\right|_{\infty},\left|W^{\delta, n}\right|_{\infty}\right\} \leq \delta
$$

then

$$
U^{\delta, n}=U^{n} \quad \text { and } \quad W^{\delta, n}=W^{n}, \quad n=1, \ldots, N .
$$

Proof. Since $\delta>\delta_{\star}$, we obtain $\left|U^{\delta, 0}\right|_{\infty}=\left|u^{0}\right|_{\infty} \leq \delta$ and $\left|W^{\delta, 0}\right|_{\infty}=\left|w^{0}\right|_{\infty} \leq \delta$. Using the latter inequalities along with the assumption (3.23) and relation (3.19), we conclude that

$$
\gamma_{\delta}\left(U^{\delta, n}\right)=U^{\delta, n} \quad \text { and } \quad \gamma_{\delta}\left(W^{\delta, n}\right)=W^{\delta, n}, \quad n=0, \ldots, N .
$$

Finally, we arrive at (3.24) using (3.25) and a simple induction argument based on (3.21) and (3.22).

\subsection{Lipschitz-type inequalities}

In this section, we show several Lipschitz-type inequalities that will serve later the error estimation of the modified finite difference method.

Lemma 3.4. Let $\varepsilon>0$ and $F_{\mathbb{C}}: \mathbb{C}^{2} \rightarrow \mathbb{R}$ defined by $F_{\mathbb{C}}(z, w):=F_{\mathbb{R}}(\operatorname{Re}(z), \operatorname{Im}(z), \operatorname{Re}(w), \operatorname{Im}(w))$ for $z, w \in \mathbb{C}$, where $F_{\mathbb{R}} \in C^{1}\left(\mathbb{R}^{4} ; \mathbb{R}\right)$. If $\left(v^{m}\right)_{m=1}^{4} \subset X_{h}$ with $\max _{1 \leq m \leq 4}\left|v^{m}\right|_{\infty} \leq \varepsilon$, then

$$
\left\|F_{\mathbb{C}}\left(v^{1}, v^{2}\right)-F_{\mathbb{C}}\left(v^{3}, v^{4}\right)\right\|_{0, h} \leq \widehat{C}_{\varepsilon}\left(\left\|v^{1}-v^{3}\right\|_{0, h}+\left\|v^{2}-v^{4}\right\|_{0, h}\right),
$$

where $\widehat{C}_{\varepsilon}=\sup _{\left(K_{\varepsilon}\right)^{4}}\left\|\nabla F_{\mathbb{R}}\right\|_{2, \mathbb{R}^{4}}$. 
Proof. Let $x^{A}, x^{B} \in \mathbb{R}^{4}$ with $\max \left\{\left\|x^{A}\right\|_{\infty, \mathbb{R}^{4}},\left\|x^{B}\right\|_{\infty, \mathbb{R}^{4}}\right\} \leq \varepsilon$. Using the Taylor formula for scalar functions with integral remainder we conclude that $F_{\mathbb{R}}\left(x^{A}\right)-F_{\mathbb{R}}\left(x^{B}\right)=\int_{0}^{1} \nabla F_{\mathbb{R}}\left(s x^{A}+(1-s) x^{B}\right)\left(x^{A}-x^{B}\right) \mathrm{d} s$, which yields that $\left|F_{\mathbb{R}}\left(x^{A}\right)-F_{\mathbb{R}}\left(x^{B}\right)\right| \leq \widehat{C}_{\varepsilon}\left\|x^{A}-x^{B}\right\|_{2, \mathbb{R}^{4}}$. Now, we combine last inequality and our assumptions on $\left(v^{m}\right)_{m=1}^{4}$ to obtain

$$
\left\|F_{\mathbb{C}}\left(v^{1}, v^{2}\right)-F_{\mathbb{C}}\left(v^{3}, v^{4}\right)\right\|_{0, h} \leq \widehat{C}_{\varepsilon}\left(h \sum_{j=1}^{J+1}\left(\left|v_{j}^{1}-v_{j}^{3}\right|^{2}+\left|v_{j}^{2}-v_{j}^{4}\right|^{2}\right)\right)^{\frac{1}{2}}
$$

which obviously yields (3.26).

Lemma 3.5. Let $\delta_{\star}$ be the constant defined by (3.16), $\delta>\delta_{\star}$ and $\gamma_{\delta}: \mathbb{C} \rightarrow \mathbb{C}$ be the function defined in (3.18). Then, it holds that

$$
\forall \omega_{A}, \omega_{B} \in X_{h}: \quad\left\|\gamma_{\delta}\left(\omega_{A}\right)-\gamma_{\delta}\left(\omega_{B}\right)\right\|_{0, h} \leq \widetilde{C}_{\delta}\left\|\omega_{A}-\omega_{B}\right\|_{0, h},
$$

where $\widetilde{C}_{\delta}=\sup _{K_{3 \delta}}\left|g_{\delta}^{\prime}\right|$.

Proof. The inequality (3.27) follows easily by using the mean-value theorem on the function $g_{\delta}$ and observing that $g_{\delta}^{\prime}(x)=0$ for $x \in \mathbb{R} \backslash K_{3 \delta}$.

Lemma 3.6. Let $\varepsilon>0$ and $F_{\mathbb{C}}: \mathbb{C}^{2} \rightarrow \mathbb{R}$ defined by $F_{\mathbb{C}}(z, w):=F_{\mathbb{R}}(\operatorname{Re}(z), \operatorname{Im}(z), \operatorname{Re}(w), \operatorname{Im}(w))$ for $z, w \in \mathbb{C}$, where $F_{\mathbb{R}} \in C^{2}\left(\mathbb{R}^{4} ; \mathbb{R}\right)$. If $\left(v^{m}\right)_{m=1}^{4} \subset X_{h}$ with $\max _{1 \leq m \leq 4}\left|v^{m}\right|_{\infty} \leq \varepsilon$, then

$$
\begin{aligned}
\left\|\delta_{h} F_{\mathbb{C}}\left(v^{1}, v^{2}\right)-\delta_{h} F_{\mathbb{C}}\left(v^{3}, v^{4}\right)\right\|_{0, h} \leq & \widehat{C}_{1, \varepsilon}\left[\left\|\delta_{h} v^{1}-\delta_{h} v^{3}\right\|_{0, h}+\left\|\delta_{h} v^{2}-\delta_{h} v^{4}\right\|_{0, h}\right. \\
& \left.+\left(\left|\delta_{h} v^{1}\right|_{\infty}+\left|\delta_{h} v^{2}\right|_{\infty}\right)\left(\left\|v^{1}-v^{3}\right\|_{0, h}+\left\|v^{2}-v^{4}\right\|_{0, h}\right)\right],
\end{aligned}
$$

with $\widehat{C}_{1, \varepsilon}=\max \left\{\sup _{\left(K_{\varepsilon}\right)^{4}}\left\|\nabla F_{\mathbb{R}}\right\|_{2, \mathbb{R}^{4}}, \max _{1 \leq j \leq 4}\left(\sup _{\left(K_{\varepsilon}\right)^{4}}\left\|\nabla \partial_{x_{j}} F_{\mathbb{R}}\right\|_{2, \mathbb{R}^{4}}\right)\right\}$.

Proof. Let $\left(x^{i}\right)_{i=1}^{4} \subset \mathbb{R}^{4}$ with $\max _{1 \leq i \leq 4}\left\|x^{i}\right\|_{\infty, \mathbb{R}^{4}} \leq \varepsilon$. Using the mean value theorem for scalar functions we have

$$
\begin{aligned}
F_{\mathbb{R}}\left(x^{1}\right)-F_{\mathbb{R}}\left(x^{2}\right)-F_{\mathbb{R}}\left(x^{3}\right)+F_{\mathbb{R}}\left(x^{4}\right)= & \int_{0}^{1} \nabla F_{\mathbb{R}}\left(s x^{3}+(1-s) x^{4}\right)\left(x^{1}-x^{2}-x^{3}+x^{4}\right) \mathrm{d} s+\sum_{j=1}^{4}\left(x_{j}^{1}-x_{j}^{2}\right) \\
& \times \int_{0}^{1} \int_{0}^{1} \nabla \partial_{x_{j}} F_{\mathbb{R}}\left(r\left(s, s^{\prime}\right)\right)\left[s\left(x^{1}-x^{3}\right)+(1-s)\left(x^{2}-x^{4}\right)\right] \mathrm{d} s \mathrm{~d} s^{\prime}
\end{aligned}
$$

where $r\left(s, s^{\prime}\right):=s s^{\prime} x^{1}+s^{\prime}(1-s) x^{2}+\left(1-s^{\prime}\right) s x^{3}+\left(1-s^{\prime}\right)(1-s) x^{4}$. Hence, we obtain

$$
\begin{aligned}
\left|F_{\mathbb{R}}\left(x^{1}\right)-F_{\mathbb{R}}\left(x^{2}\right)-F_{\mathbb{R}}\left(x^{3}\right)+F_{\mathbb{R}}\left(x^{4}\right)\right| \leq & \widehat{C}_{1, \varepsilon}\left[\left\|\left(x^{1}-x^{2}\right)-\left(x^{3}-x^{4}\right)\right\|_{2, \mathbb{R}^{4}}\right. \\
& \left.+\frac{1}{2}\left\|x^{1}-x^{2}\right\|_{1, \mathbb{R}^{4}}\left(\left\|x^{1}-x^{3}\right\|_{2, \mathbb{R}^{4}}+\left\|x^{2}-x^{4}\right\|_{2, \mathbb{R}^{4}}\right)\right] .
\end{aligned}
$$

Using the inequality above, we get

$$
\begin{aligned}
\left\|\delta_{h} F_{\mathbb{C}}\left(v^{1}, v^{2}\right)-\delta_{h} F_{\mathbb{C}}\left(v^{3}, v^{4}\right)\right\|_{0, h} \leq & \widehat{C}_{1, \varepsilon}\left\{\left[h \sum_{j=1}^{J+1}\left(\left|\delta_{h} v_{j}^{1}-\delta_{h} v_{j}^{3}\right|^{2}+\left|\delta_{h} v_{j}^{2}-\delta_{h} v_{j}^{4}\right|^{2}\right)\right]^{\frac{1}{2}}\right. \\
& +\left[h \sum_{j=1}^{J+1}\left(\left|\delta_{h} v_{j}^{1}\right|+\left|\delta_{h} v_{j}^{2}\right|\right)^{2}\left(\left|v_{j+1}^{1}-v_{j+1}^{3}\right|^{2}+\left|v_{j+1}^{2}-v_{j+1}^{4}\right|^{2}\right)\right]^{\frac{1}{2}} \\
& \left.+\left[h \sum_{j=1}^{J+1}\left(\left|\delta_{h} v_{j}^{1}\right|+\left|\delta_{h} v_{j}^{2}\right|\right)^{2}\left(\left|v_{j-1}^{1}-v_{j-1}^{3}\right|^{2}+\left|v_{j-1}^{2}-v_{j-1}^{4}\right|^{2}\right)\right]^{\frac{1}{2}}\right\}
\end{aligned}
$$

which, easily, yields (3.28). 
Lemma 3.7. Let $\delta_{\star}$ be the constant defined by (3.16), $\delta>\delta_{\star}$ and $\gamma_{\delta}: \mathbb{C} \rightarrow \mathbb{C}$ be the function defined in (3.18). Then, it holds that

$$
\forall \omega, v \in X_{h}: \quad\left\|\delta_{h} \gamma_{\delta}(\omega)-\delta_{h} \gamma_{\delta}(v)\right\|_{0, h} \leq \widetilde{C}_{1, \delta}\left(\left\|\delta_{h} \omega-\delta_{h} v\right\|_{0, h}+\left|\delta_{h} \omega\right|_{\infty}\|\omega-v\|_{0, h}\right),
$$

where $\widetilde{C}_{1, \delta}:=\sqrt{2} \max \left\{\sup _{K_{3 \delta}}\left|g_{\delta}^{\prime}\right|, \sup _{K_{3 \delta}}\left|g_{\delta}^{\prime \prime}\right|\right\}$.

Proof. Proceeding as in the proof of Lemma 3.6 we arrive at the following inequality

$$
\left|g_{\delta}\left(x_{1}\right)-g_{\delta}\left(x_{2}\right)-g_{\delta}\left(x_{3}\right)+g_{\delta}\left(x_{4}\right)\right| \leq \frac{\widetilde{C}_{1, \delta}}{\sqrt{2}}\left[\left|x_{1}-x_{2}-x_{3}+x_{4}\right|+\frac{1}{2}\left|x_{1}-x_{2}\right|\left(\left|x_{1}-x_{3}\right|+\left|x_{2}-x_{4}\right|\right)\right]
$$

for $x_{1}, x_{2}, x_{3}, x_{4} \in \mathbb{R}$. Applying the latter inequality, we obtain

$$
\begin{aligned}
\left\|\delta_{h} \gamma_{\delta}(\omega)-\delta_{h} \gamma_{\delta}(v)\right\|_{0, h} \leq & \widetilde{C}_{1, \delta}\left[\left(h \sum_{j=1}^{J+1}\left|\delta_{h} \omega_{j}-\delta_{h} v_{j}\right|^{2}\right)^{\frac{1}{2}}\right. \\
& \left.+\frac{1}{2}\left(\sum_{j=1}^{J+1}\left|\delta_{h} \omega_{j}\right|^{2}\left[\left|\omega_{j+1}-v_{j+1}\right|+\left|\omega_{j-1}-v_{j-1}\right|\right]^{2}\right)^{\frac{1}{2}}\right]
\end{aligned}
$$

that easily yields (3.29).

\subsection{Convergence analysis}

As a first step of our analysis, we prove an optimal order convergence result, in the norm $\|\cdot\|_{0, h}$, for the approximations of the modified finite difference method.

Proposition 3.8. Let $\delta_{\star}$ be the constant defined by $(3.16), \delta>\delta_{\star},\left\{\left(U^{\delta, m}, W^{\delta, m}\right)\right\}_{m=0}^{N}$ be the approximations specified by the modified finite difference method (3.20)-(3.22) and $\left\{\left(u^{m}, w^{m}\right)\right\}_{m=0}^{N}$ be defined by (3.1). Then we have

$$
\max _{0 \leq n \leq N}\left(\left\|U^{\delta, n}-u^{n}\right\|_{0, h}+\left\|W^{\delta, n}-w^{n}\right\|_{0, h}\right) \leq C_{0, \delta}\left(\tau^{2}+h^{2}\right)
$$

where $C_{0, \delta}$ is a constant depending on $\delta$, but independent of $\tau$ and $h$.

Proof. First, we set $e_{U}^{\delta, m}:=u^{m}-U^{\delta, m}$ and $e_{W}^{\delta, m}:=w^{m}-W^{\delta, m}$ for $m=0, \ldots, N$. Then, we introduce $\left(\mu^{\delta, m}\right)_{m=1}^{N} \subset X_{h}$ and $\left(\xi^{\delta, m}\right)_{m=1}^{N} \subset X_{h}$, by

$$
\begin{aligned}
& \partial_{\tau}^{\mathrm{init}} e_{U}^{\delta, 1}+\delta_{h} \mathcal{A}^{\mathrm{init}} e_{W}^{\delta, 1}=i \alpha_{1} \mathcal{A}^{\mathrm{init}} e_{U}^{\delta, 1}+\mu^{\delta, 1}+\rho^{1}, \\
& \partial_{\tau}^{\mathrm{init}} e_{W}^{\delta, 1}+\delta_{h} \mathcal{A}^{\mathrm{init}} e_{U}^{\delta, 1}=i \alpha_{2} \mathcal{A}^{\mathrm{init}} e_{W}^{\delta, 1}+\xi^{\delta, 1}+\sigma^{1},
\end{aligned}
$$

and

$$
\begin{aligned}
& \partial_{\tau} e_{U}^{\delta, n}+\delta_{h} \mathcal{A} e_{W}^{\delta, n}=i \alpha_{1} \mathcal{A} e_{U}^{\delta, n}+\mu^{\delta, n}+\rho^{n}, \\
& \partial_{\tau} e_{W}^{\delta, n}+\delta_{h} \mathcal{A} e_{U}^{\delta, n}=i \alpha_{2} \mathcal{A} e_{W}^{\delta, n}+\xi^{\delta, n}+\sigma^{n}
\end{aligned}
$$

for $n=1, \ldots, N$, where $\left(\rho^{n}\right)_{n=1}^{N}$ and $\left(\sigma^{n}\right)_{n=1}^{N}$ are the consistency errors defined by (3.13)-(3.14). Subtracting (3.21) from (3.13), and (3.22) from (3.14), we easily verify that

$$
\begin{aligned}
& \mu^{\delta, n}=-i \lambda_{1}\left[f_{\delta}\left(U^{\delta, n-1}, W^{\delta, n-1}\right) \otimes \gamma_{\delta}\left(U^{\delta, n-1}\right)-f_{\delta}\left(u^{n-1}, w^{n-1}\right) \otimes \gamma_{\delta}\left(u^{n-1}\right)\right], \\
& \xi^{\delta, n}=-i \lambda_{2}\left[f_{\delta}\left(U^{\delta, n-1}, W^{\delta, n-1}\right) \otimes \gamma_{\delta}\left(W^{\delta, n-1}\right)-f_{\delta}\left(u^{n-1}, w^{n-1}\right) \otimes \gamma_{\delta}\left(w^{n-1}\right)\right]
\end{aligned}
$$


for $n=1, \ldots, N$. Also, we observe that (3.20) yields

$$
e_{U}^{\delta, 0}=0, \quad e_{W}^{\delta, 0}=0 \quad \text { and } \mu^{\delta, 1}=0, \quad \xi^{\delta, 1}=0 .
$$

Now, take the $(\cdot, \cdot)_{0, h}$ inner product of $(3.31 \mathrm{a})$ by $\mathcal{A}^{\mathrm{init}} e_{U}^{\delta, 1}$ and of $(3.31 \mathrm{~b})$ by $\mathcal{A}^{\mathrm{init}} e_{W}^{\delta, 1}$, and then real parts. We add the obtained relations, and then we use (3.2), the consistency estimate (3.15a) and (3.34) to obtain

$$
\left\|e_{U}^{\delta, 1}\right\|_{0, h}+\left\|e_{W}^{\delta, 1}\right\|_{0, h} \leq C\left(\tau^{2}+\tau h^{2}\right) .
$$

Next, set

$$
\nu^{\delta, m}:=\left(\left\|e_{U}^{\delta, m}\right\|_{0, h}^{2}+\left\|e_{W}^{\delta, m}\right\|_{0, h}^{2}+\left\|e_{U}^{\delta, m-1}\right\|_{0, h}^{2}+\left\|e_{W}^{\delta, m-1}\right\|_{0, h}^{2}\right)^{\frac{1}{2}}, \quad m=1, \ldots, N,
$$

take the $(\cdot, \cdot)_{0, h}$ inner product of $(3.32 \mathrm{a})$ by $\mathcal{A} e_{U}^{\delta, n}$ and of $(3.32 \mathrm{~b})$ by $\mathcal{A} e_{W}^{\delta, n}$, and then real parts. We add the obtained relations, and then we use, again, (3.2), the consistency estimate (3.15a) and (3.34) to obtain

$$
\begin{aligned}
\left(\nu^{\delta, n}\right)^{2} \leq & \left(\nu^{\delta, n-1}\right)^{2}+2 \tau\left\|\mu^{\delta, n}\right\|_{0, h}\left(\nu^{\delta, n}+\nu^{\delta, n-1}\right)+2 \tau\left\|\xi^{\delta, n}\right\|_{0, h}\left(\nu^{\delta, n}+\nu^{\delta, n-1}\right) \\
& +C \tau\left(\tau^{2}+h^{2}\right)\left(\nu^{\delta, n}+\nu^{\delta, n-1}\right), \quad n=2, \ldots, N .
\end{aligned}
$$

Let $n \in\{2, \ldots, N\}$. Our next step is to estimate the quantities $\left\|\mu^{\delta, n}\right\|_{0, h}$ and $\left\|\xi^{\delta, n}\right\|_{0, h}$. First we observe that

$$
\left\|\mu^{\delta, n}\right\|_{0, h} \leq\left|\lambda_{1}\right|\left(E_{\mu, 1}^{n}+E_{\mu, 2}^{n}\right),
$$

where

$$
E_{\mu, 1}^{n}:=\left\|\left[f_{\delta}\left(U^{\delta, n-1}, W^{\delta, n-1}\right)-f_{\delta}\left(u^{n-1}, w^{n-1}\right)\right] \otimes \gamma_{\delta}\left(U^{\delta, n-1}\right)\right\|_{0, h}
$$

and

$$
E_{\mu, 2}^{n}:=\left\|f\left(u^{n-1}, w^{n-1}\right) \otimes\left[\gamma_{\delta}\left(U^{\delta, n-1}\right)-\gamma_{\delta}\left(u^{n-1}\right)\right]\right\|_{0, h} .
$$

Then, using the properties of $g_{\delta}$, Lemmas 3.4 and 3.5 , we obtain

$$
\begin{aligned}
E_{\mu, 1}^{n} & \leq\left|\gamma_{\delta}\left(U^{\delta, n-1}\right)\right|_{\infty}\left\|f\left(\gamma_{\delta}\left(U^{\delta, n-1}\right), \gamma_{\delta}\left(W^{\delta, n-1}\right)\right)-f\left(\gamma_{\delta}\left(u^{n-1}\right), \gamma_{\delta}\left(w^{n-1}\right)\right)\right\|_{0, h} \\
& \leq 2 \sqrt{2} \delta\left\|f\left(\gamma_{\delta}\left(U^{\delta, n-1}\right), \gamma_{\delta}\left(W^{\delta, n-1}\right)\right)-f\left(\gamma_{\delta}\left(u^{n-1}\right), \gamma_{\delta}\left(w^{n-1}\right)\right)\right\|_{0, h} \\
& \leq C_{\delta} \quad\left(\left\|\gamma_{\delta}\left(U^{\delta, n-1}\right)-\gamma_{\delta}\left(u^{n-1}\right)\right\|_{0, h}+\left\|\gamma_{\delta}\left(W^{\delta, n-1}\right)-\gamma_{\delta}\left(w^{n-1}\right)\right\|_{0, h}\right) \\
& \leq C_{\delta}\left(\left\|e_{U}^{\delta, n-1}\right\|_{0, h}+\left\|e_{W}^{\delta, n-1}\right\|_{0, h}\right)
\end{aligned}
$$

and

$$
\begin{aligned}
E_{\mu, 2}^{n} & \leq\left|f\left(u^{n-1}, w^{n-1}\right)\right|_{\infty}\left\|\gamma_{\delta}\left(U^{\delta, n-1}\right)-\gamma_{\delta}\left(u^{n-1}\right)\right\|_{0, h} \\
& \leq \sup _{\left(K_{\delta_{\star}}\right)^{4}}\left|f_{\mathbb{R}}\right|\left\|\gamma_{\delta}\left(U^{\delta, n-1}\right)-\gamma_{\delta}\left(u^{n-1}\right)\right\|_{0, h} \\
& \leq C_{\delta}\left\|e_{U}^{\delta, n-1}\right\|_{0, h} .
\end{aligned}
$$

Thus, combining (3.37)-(3.39), it follows that

$$
\begin{aligned}
\left\|\mu^{\delta, n}\right\|_{0, h} & \leq C_{\delta}\left(\left\|e_{U}^{\delta, n-1}\right\|_{0, h}+\left\|e_{W}^{\delta, n-1}\right\|_{0, h}\right) \\
& \leq C_{\delta} \nu^{\delta, n-1} .
\end{aligned}
$$


Finally, to estimate $\left\|\xi^{\delta, n}\right\|_{0, h}$, we proceed in a similar way to get

$$
\begin{aligned}
\left\|\xi^{\delta, n}\right\|_{0, h} & \leq C_{\delta}\left(\left\|e_{U}^{\delta, n-1}\right\|_{0, h}+\left\|e_{W}^{\delta, n-1}\right\|_{0, h}\right) \\
& \leq C_{\delta} \nu^{\delta, n-1} .
\end{aligned}
$$

Combining (3.36), (3.40) and (3.41), we get

$$
\nu^{\delta, n} \leq\left(1+C_{\delta} \tau\right) \nu^{\delta, n-1}+C \tau\left(\tau^{2}+h^{2}\right), \quad n=2, \ldots, N .
$$

Then, a standard discrete Grönwall argument yields

$$
\max _{1 \leq n \leq N} \nu^{\delta, n} \leq C_{\delta}\left(\nu^{\delta, 1}+\tau^{2}+h^{2}\right) .
$$

Finally, the estimate (3.30) follows combining (3.42), (3.35) and (3.34).

Next we shall show that the approximations derived by the modified finite difference method are bounded, in the seminorm $|\cdot|_{1, h}$, by a constant which is independent of $\tau$ and $h$.

Proposition 3.9. Let $\delta_{\star}$ be the constant defined by (3.16), $\delta>\delta_{\star}$ and $\left\{\left(U^{\delta, m}, W^{\delta, m}\right)\right\}_{m=0}^{N}$ be the approximations of the modified finite difference method (3.20)-(3.22). Then, we have

$$
\max _{0 \leq n \leq N}\left(\left|U^{\delta, n}\right|_{1, h}+\left|W^{\delta, n}\right|_{1, h}\right) \leq C_{B, \delta}
$$

where $C_{B, \delta}$ is a constant depending on $\delta$, but independent of $\tau$ and $h$.

Proof. First we set $U_{\star}^{\delta, m}:=\delta_{h} U^{\delta, m}$ and $W_{\star}^{\delta, m}:=\delta_{h} W^{\delta, m}$ for $m=0, \ldots, N$. Now, we apply the operator $\delta_{h}$ on (3.21) and (3.22) to obtain

$$
\begin{aligned}
& \partial_{\tau}^{\text {init }} U_{\star}^{\delta, 1}+\delta_{h} \mathcal{A}^{\text {init }} W_{\star}^{\delta, 1}=i \alpha_{1} \mathcal{A}^{\text {init }} U_{\star}^{\delta, 1}+\mu_{\star}^{\delta, 1}, \\
& \partial_{\tau}^{\text {init }} W_{\star}^{\delta, 1}+\delta_{h} \mathcal{A}^{\text {init }} U_{\star}^{\delta, 1}=i \alpha_{2} \mathcal{A}^{\text {init }} W_{\star}^{\delta, 1}+\xi_{\star}^{\delta, 1},
\end{aligned}
$$

and

$$
\begin{aligned}
& \partial_{\tau} U_{\star}^{\delta, n}+\delta_{h} \mathcal{A} W_{\star}^{\delta, n}=i \alpha_{1} \mathcal{A} U_{\star}^{\delta, n}+\mu_{\star}^{\delta, n}, \\
& \partial_{\tau} W_{\star}^{\delta, n}+\delta_{h} \mathcal{A} U_{\star}^{\delta, n}=i \alpha_{2} \mathcal{A} W_{\star}^{\delta, n}+\xi_{\star}^{\delta, n}, \quad n=2, \ldots, N,
\end{aligned}
$$

where

$$
\begin{aligned}
& \mu_{\star}^{\delta, n}:=i \lambda_{1} \delta_{h}\left[f_{\delta}\left(U^{\delta, n-1}, W^{\delta, n-1}\right) \otimes \gamma_{\delta}\left(U^{\delta, n-1}\right)\right] \\
& \xi_{\star}^{\delta, n}:=i \lambda_{2} \delta_{h}\left[f_{\delta}\left(U^{\delta, n-1}, W^{\delta, n-1}\right) \otimes \gamma_{\delta}\left(W^{\delta, n-1}\right)\right] .
\end{aligned}
$$

Now, letting

$$
\pi_{\star}^{\delta, m}:=\left(\left\|U_{\star}^{\delta, m}\right\|_{0, h}^{2}+\left\|W_{\star}^{\delta, m}\right\|_{0, h}^{2}+\left\|U_{\star}^{\delta, m-1}\right\|_{0, h}^{2}+\left\|W_{\star}^{\delta, m-1}\right\|_{0, h}^{2}\right)^{\frac{1}{2}}, \quad m=1, \ldots, N,
$$

and proceeding as in the proof of Proposition 3.8 we arrive at

$$
\left(\left\|U_{\star}^{\delta, 1}\right\|_{0, h}^{2}+\left\|W_{\star}^{\delta, 1}\right\|_{0, h}^{2}\right)^{\frac{1}{2}} \leq\left(\left\|\delta_{h} u^{0}\right\|_{0, h}^{2}+\left\|\delta_{h} w^{0}\right\|_{0, h}^{2}\right)^{\frac{1}{2}}+\tau\left(\left\|\mu_{\star}^{\delta, 1}\right\|_{0, h}+\left\|\xi_{\star}^{\delta, 1}\right\|_{0, h}\right)
$$

and

$$
\pi_{\star}^{\delta, n} \leq \pi_{\star}^{\delta, n-1}+2 \tau\left(\left\|\mu_{\star}^{\delta, n}\right\|_{0, h}+\left\|\xi_{\star}^{\delta, n}\right\|_{0, h}\right), \quad n=2, \ldots, N .
$$


Let $n \in\{1, \ldots, N\}$. To derive a bound for $\left\|\mu_{\star}^{\delta, n}\right\|_{0, h}$, we use (3.3) to split it as follows

$$
\left\|\mu_{\star}^{\delta, n}\right\|_{0, h} \leq\left|\lambda_{1}\right|\left(M_{1}^{\delta, n}+M_{2}^{\delta, n}\right),
$$

where

$$
\begin{aligned}
& M_{1}^{\delta, n}:=\left\|\theta_{h}^{+}\left(f\left(\gamma_{\delta}\left(U^{\delta, n-1}\right), \gamma_{\delta}\left(W^{\delta, n-1}\right)\right)\right) \otimes \delta_{h}\left(\gamma_{\delta}\left(U^{\delta, n-1}\right)\right)\right\|_{0, h}, \\
& M_{2}^{\delta, n}:=\left\|\delta_{h}\left(f\left(\gamma_{\delta}\left(U^{\delta, n-1}\right), \gamma_{\delta}\left(W^{\delta, n-1}\right)\right)\right) \otimes \theta_{h}^{-}\left(\gamma_{\delta}\left(U^{\delta, n-1}\right)\right)\right\|_{0, h} .
\end{aligned}
$$

Using Lemmas 3.7 and 3.6, we obtain

$$
\begin{aligned}
M_{1}^{\delta, n} & \leq\left|f\left(\gamma_{\delta}\left(U^{\delta, n-1}\right), \gamma_{\delta}\left(W^{\delta, n-1}\right)\right)\right|_{\infty}\left\|\delta_{h} \gamma_{\delta}\left(U^{\delta, n-1}\right)\right\|_{0, h} \\
& \leq \sup _{\left(K_{2 \sqrt{2} \delta}\right)^{4}}\left|f_{\mathbb{R}}\right|\left\|\delta_{h} \gamma_{\delta}(0)-\delta_{h} \gamma_{\delta}\left(U^{\delta, n-1}\right)\right\|_{0, h} \\
& \leq C_{\delta}\left\|U_{\star}^{\delta, n-1}\right\|_{0, h}
\end{aligned}
$$

and

$$
\begin{aligned}
M_{2}^{\delta, n} & \leq\left|\gamma_{\delta}\left(U^{\delta, n-1}\right)\right|_{\infty}\left\|\delta_{h} f\left(\gamma_{\delta}\left(U^{\delta, n-1}\right), \gamma_{\delta}\left(W^{\delta, n-1}\right)\right)\right\|_{0, h} \\
& \leq 2 \sqrt{2} \delta\left\|\delta_{h} f\left(\gamma_{\delta}(0), \gamma_{\delta}(0)\right)-\delta_{h} f\left(\gamma_{\delta}\left(U^{\delta, n-1}\right), \gamma_{\delta}\left(W^{\delta, n-1}\right)\right)\right\|_{0, h} \\
& \leq C_{\delta}\left(\left\|\delta_{h} \gamma_{\delta}\left(U^{\delta, n-1}\right)\right\|_{0, h}+\left\|\delta_{h} \gamma_{\delta}\left(W^{\delta, n-1}\right)\right\|_{0, h}\right) \\
& \leq C_{\delta}\left(\left\|U_{\star}^{\delta, n-1}\right\|_{0, h}+\left\|W_{\star}^{\delta, n-1}\right\|_{0, h}\right) .
\end{aligned}
$$

Hence, (3.46)-(3.48) yield

$$
\left\|\mu_{\star}^{\delta, n}\right\|_{0, h} \leq C_{\delta}\left(\left\|U_{\star}^{\delta, n-1}\right\|_{0, h}+\left\|W_{\star}^{\delta, n-1}\right\|_{0, h}\right) .
$$

Proceeding in a similar way we get

$$
\left\|\xi_{\star}^{\delta, n}\right\|_{0, h} \leq C_{\delta}\left(\left\|U_{\star}^{\delta, n-1}\right\|_{0, h}+\left\|W_{\star}^{\delta, n-1}\right\|_{0, h}\right) .
$$

From (3.44), (3.49) and (3.50) we obtain

$$
\pi_{\star}^{\delta, 1} \leq C_{\delta}\left(\left\|\delta_{h} u^{0}\right\|_{0, h}+\left\|\delta_{h} w^{0}\right\|_{0, h}\right) .
$$

Combining (3.45), (3.49) and (3.50) we get

$$
\pi_{\star}^{\delta, n} \leq\left(1+C_{\delta} \tau\right) \pi_{\star}^{\delta, n-1}, \quad n=2, \ldots, N,
$$

which, following a standard discrete Grönwall induction argument, yields

$$
\max _{1 \leq n \leq N} \pi_{\star}^{\delta, n} \leq C_{\delta} \pi_{\star}^{\delta, 1} .
$$

Observing that $\left\|\delta_{h} u^{0}\right\|_{0, h}+\left\|\delta_{h} w^{0}\right\|_{0, h}=\mathcal{O}(1)$, the bound (3.43) follows as a simple consequence of (3.51) and (3.52) .

We are ready to prove an optimal order error estimate, in the norm $\|\cdot\|_{0, h}$, for the approximations of the finite difference method described in Section 1.3.

Theorem 3.10. Let $\delta_{\star}$ be the constant defined by (3.16), $\delta_{0}>2 \delta_{\star},\left\{\left(U^{m}, W^{m}\right)\right\}_{m=0}^{N}$ be the approximations of the finite difference method (3.7)-(3.9), $C_{S}$ be the constant in the inequality (3.4), $C_{0, \delta_{0}}$ be the constant in the inequality (3.30) for $\delta=\delta_{0}, C_{B, \delta_{0}}$ be the constant in the inequality (3.43) for $\delta=\delta_{0}$, and $c_{\star}:=$ $\max \left\{\sup _{[0, T]}\left\|u_{x}\right\|_{L^{\infty}(0, L)}, \sup _{[0, T]}\left\|w_{x}\right\|_{L^{\infty}(0, L)}\right\}$. If $\tau$ and $h$ are small enough such that

$$
\sqrt{\tau^{2}+h^{2}} \sqrt{C_{S} C_{0, \delta_{0}}}\left[C_{0, \delta_{0}}\left(\tau^{2}+h^{2}\right)+C_{B, \delta_{0}}+c_{\star} \sqrt{L}\right]^{\frac{1}{2}} \leq \delta_{\star}
$$


then there exists a constant $C>0$ independent of $\tau$ and $h$, such that

$$
\max _{0 \leq n \leq N}\left(\left\|U^{n}-u^{n}\right\|_{0, h}+\left\|W^{n}-w^{n}\right\|_{0, h}\right) \leq C\left(\tau^{2}+h^{2}\right) .
$$

Proof. Let $\left\{\left(U^{\delta_{0}, m}, W^{\delta_{0}, m}\right)\right\}_{m=0}^{N}$ be the approximations of the modified finite difference method (3.20)-(3.22) for $\delta=\delta_{0}$. Then, using (3.4), (3.30), (3.43) and (3.53), we obtain

$$
\begin{aligned}
\left|U^{\delta_{0}, n}\right|_{\infty} & \leq\left|u^{n}\right|_{\infty}+\left|U^{\delta_{0}, n}-u^{n}\right|_{\infty} \\
& \leq \delta_{\star}+\sqrt{C_{S}}\left\|U^{\delta_{0}, n}-u^{n}\right\|_{0, h}^{\frac{1}{2}}\left(\left\|U^{\delta_{0}, n}-u^{n}\right\|_{0, h}+\left|U^{\delta_{0}, n}-u^{n}\right|_{1, h}\right)^{\frac{1}{2}} \\
& \leq \delta_{\star}+\sqrt{C_{S} C_{0, \delta_{0}}}\left(\tau^{2}+h^{2}\right)^{\frac{1}{2}}\left(C_{0, \delta_{0}}\left(\tau^{2}+h^{2}\right)+C_{B, \delta_{0}}+\left|u^{n}\right|_{1, h}\right)^{\frac{1}{2}} \\
& \leq \delta_{\star}+\sqrt{C_{S} C_{0, \delta_{0}}}\left(\tau^{2}+h^{2}\right)^{\frac{1}{2}}\left[C_{0, \delta_{0}}\left(\tau^{2}+h^{2}\right)+C_{B, \delta_{0}}+\sqrt{L}\left\|u_{x}\left(\cdot, t^{n}\right)\right\|_{L_{\infty}^{\infty}(0, L)}\right]^{\frac{1}{2}} \\
& \leq 2 \delta_{\star} \\
& \leq \delta_{0}, \quad n=1, \ldots, N .
\end{aligned}
$$

Proceeding in a analogous way, we, also, obtain that $\left|W^{\delta_{0}, n}\right|_{\infty} \leq \delta_{0}$ for $n=1, \ldots, N$. Thus, according to Lemma 3.3, we conclude that $U^{\delta_{0}, n}=U^{n}$ and $W^{\delta_{0}, n}=W^{n}$ for $n=1, \ldots, N$, and the error estimate (3.54) follows from (3.30) and (3.7).

Remark 3.11. Let us assume that the a priori bound (3.43) is not available. Using the inverse inequality $|v|_{\infty} \leq h^{-\frac{1}{2}}\|v\|_{0, h}$ for $v \in X_{h}$, and the error estimate (3.30), we arrive at

$$
\max _{0 \leq n \leq N}\left(\left|U^{\delta_{0}, n}\right|_{\infty}+\left|W^{\delta_{0}, n}\right|_{\infty}\right) \leq \delta_{\star}+C_{0, \delta_{0}}\left(\tau^{2} h^{-\frac{1}{2}}+h^{\frac{3}{2}}\right)
$$

Requiring $\delta_{0}>2 \delta_{\star}$ and $\tau=o\left(h^{-\frac{1}{4}}\right)$, we can have $C_{0, \delta_{0}}\left(\tau^{2} h^{-\frac{1}{2}}+h^{\frac{3}{2}}\right)<\delta_{\star}$ for $\tau$ and $h$ enough small, and thus we obtain $\max _{0 \leq n \leq N}\left(\left|U^{\delta_{0}, n}\right|_{\infty}+\left|W^{\delta_{0}, n}\right|_{\infty}\right) \leq \delta_{0}$ which, along with Lemma 3.3, establishes (3.54).

Our next step is to prove an optimal order error estimate, in the seminorm $|\cdot|_{1, h}$, for the approximations derived by the modified finite difference method.

Proposition 3.12. Let $\delta_{\star}$ be the constant defined by (3.16), $\delta>\delta_{\star},\left\{\left(U^{\delta, m}, W^{\delta, m}\right)\right\}_{m=0}^{N}$ be the approximations of the modified finite difference method (3.20)-(3.22) and $\left\{\left(u^{m}, w^{m}\right)\right\}_{m=0}^{N}$ be defined by (3.1). Then we have

$$
\max _{0 \leq n \leq N}\left(\left|U^{\delta, n}-u^{n}\right|_{1, h}+\left|W^{\delta, n}-w^{n}\right|_{1, h}\right) \leq C_{1, \delta}\left(\tau^{2}+h^{2}\right),
$$

where $C_{1, \delta}$ is a constant depending on $\delta$, but independent of $\tau$ and $h$.

Proof. For $m=0, \ldots, N$, we set $e_{U, \star}^{\delta, m}:=\delta_{h} e_{U}^{\delta, m}$ and $e_{W, \star}^{\delta, m}:=\delta_{h} e_{W}^{\delta, m}$, where $e_{U}^{\delta, m}$ and $e_{W}^{\delta, m}$ are the errors defined in the proof of Proposition 3.8. To construct the corresponding error equations, we apply the operator $\delta_{h}$ on the error equations (3.31a)-(3.31b) and (3.32a)-(3.32b) to obtain

$$
\begin{aligned}
& \partial_{\tau}^{\text {init }} e_{U, \star}^{\delta, 1}+\delta_{h} \mathcal{A}^{\text {init }} e_{W, \star}^{\delta, 1}=i \alpha_{1} \mathcal{A}^{\text {init }} e_{U, \star}^{\delta, 1}+\delta_{h} \mu^{\delta, 1}+\delta_{h} \rho^{1}, \\
& \partial_{\tau}^{\text {init }} e_{W, \star}^{\delta, 1}+\delta_{h} \mathcal{A}^{\text {init }} e_{U, \star}^{\delta, n}=i \alpha_{2} \mathcal{A}^{\text {init }} e_{W, \star}^{\delta, 1}+\delta_{h} \xi^{\delta, 1}+\delta_{h} \sigma^{1},
\end{aligned}
$$

and

$$
\begin{aligned}
& \partial_{\tau} e_{U, \star}^{\delta, n}+\delta_{h} \mathcal{A} e_{W, \star}^{\delta, n}=i \alpha_{1} \mathcal{A} e_{U, \star}^{\delta, n}+\delta_{h} \mu^{\delta, n}+\delta_{h} \rho^{n} \\
& \partial_{\tau} e_{W, \star}^{\delta, n}+\delta_{h} \mathcal{A}^{*} e_{U, \star}^{\delta, n}=i \alpha_{2} \mathcal{A} e_{W, \star}^{\delta, n}+\delta_{h} \xi^{\delta, n}+\delta_{h} \sigma^{n}
\end{aligned}
$$


for $n=2, \ldots, N$. Setting

$$
\nu_{\star}^{\delta, m}:=\left(\left\|e_{U, \star}^{\delta, m}\right\|_{0, h}^{2}+\left\|e_{W, \star}^{\delta, m}\right\|_{0, h}^{2}+\left\|e_{U, \star}^{\delta, m-1}\right\|_{0, h}^{2}+\left\|e_{W, \star}^{\delta, m-1}\right\|_{0, h}^{2}\right)^{\frac{1}{2}}, \quad m=1, \ldots, N,
$$

moving along the lines of the proof of Proposition 3.8 and using $(3.15 \mathrm{~b})$, we arrive at

$$
\left\|e_{U, \star}^{\delta, 1}\right\|_{0, h}+\left\|e_{W, \star}^{\delta, 1}\right\|_{0, h} \leq C\left(\tau^{2}+\tau h^{2}\right)
$$

and

$$
\nu_{\star}^{\delta, n} \leq \nu_{\star}^{\delta, n-1}+2 \tau\left(\left\|\delta_{h} \mu^{\delta, n}\right\|_{0, h}+\left\|\delta_{h} \xi^{\delta, n}\right\|_{0, h}\right)+C \tau\left(\tau^{2}+h^{2}\right), \quad n=2, \ldots, N,
$$

where we have used, also, the fact that (3.34) yields

$$
e_{U, \star}^{\delta, 0}:=0, \quad e_{W, \star}^{\delta, 0}:=0 \quad \text { and } \quad \delta_{h} \mu^{\delta, 1}=0, \quad \delta_{h} \xi^{\delta, 1}=0 .
$$

Let $n \in\{2, \ldots, N\}$. To estimate $\delta_{h} \mu^{\delta, n}$, we use (3.33) and (3.3) to introduce the following splitting

$$
\delta_{h} \mu^{\delta, n}:=-i \lambda_{1} \sum_{\ell=1}^{4} M^{n, \ell},
$$

where

$$
\begin{aligned}
& M^{n, 1}:=\theta_{h}^{+}\left(\gamma_{\delta}\left(U^{\delta, n-1}\right)\right) \otimes\left[\delta_{h} f_{\delta}\left(U^{\delta, n-1}, W^{\delta, n-1}\right)-\delta_{h} f_{\delta}\left(u^{n-1}, w^{n-1}\right)\right], \\
& M^{n, 2}:=\delta_{h} f\left(u^{n-1}, w^{n-1}\right) \otimes \theta_{h}^{+}\left(\gamma_{\delta}\left(U^{\delta, n-1}\right)-\gamma_{\delta}\left(u^{n-1}\right)\right) \\
& M^{n, 3}:=\theta_{h}^{-}\left(f_{\delta}\left(U^{\delta, n-1}, W^{\delta, n-1}\right)\right) \otimes\left[\delta_{h} \gamma_{\delta}\left(U^{\delta, n-1}\right)-\delta_{h} \gamma_{\delta}\left(u^{n-1}\right)\right] \\
& M^{n, 4}:=\theta_{h}^{-}\left(f_{\delta}\left(U^{\delta, n-1}, W^{\delta, n-1}\right)-f_{\delta}\left(u^{n-1}, w^{n-1}\right)\right) \otimes \delta_{h} u^{n-1} .
\end{aligned}
$$

Since $\max _{1 \leq n \leq N}\left|\delta_{h} u^{n-1}\right|_{\infty}=\mathcal{O}(1)$, using Lemmas 3.4-3.7 and (3.30), we obtain

$$
\begin{aligned}
& \left\|M^{n, 4}\right\|_{0, h} \leq C\left|\delta_{h} u^{n-1}\right|_{\infty}\left\|f_{\delta}\left(U^{\delta, n-1}, W^{\delta, n-1}\right)-f_{\delta}\left(u^{n-1}, w^{n-1}\right)\right\|_{0, h} \\
& \leq C\left\|f\left(\gamma_{\delta}\left(U^{\delta, n-1}\right), \gamma_{\delta}\left(W^{\delta, n-1}\right)\right)-f\left(\gamma_{\delta}\left(u^{n-1}\right), \gamma_{\delta}\left(w^{n-1}\right)\right)\right\|_{0, h} \\
& \left.\leq C_{\delta}\left[\left\|\gamma_{\delta}\left(U^{\delta, n-1}\right)-\gamma_{\delta}\left(u^{n-1}\right)\right\|_{0, h}+\| \gamma_{\delta}\left(W^{\delta, n-1}\right)\right)-\gamma_{\delta}\left(w^{n-1}\right) \|_{0, h}\right] \\
& \leq C_{\delta}\left(\left\|e_{U}^{\delta, n-1}\right\|_{0, h}+\left\|e_{W}^{\delta, n-1}\right\|_{0, h}\right) \\
& \leq C_{\delta}\left(\tau^{2}+h^{2}\right) \\
& \left\|M^{n, 3}\right\|_{0, h} \leq\left|f\left(\gamma_{\delta}\left(U^{\delta, n-1}\right), \gamma_{\delta}\left(W^{\delta, n-1}\right)\right)\right|_{\infty}\left\|\delta_{h} \gamma_{\delta}\left(U^{\delta, n-1}\right)-\delta_{h} \gamma_{\delta}\left(u^{n-1}\right)\right\|_{0, h} \\
& \leq \sup _{\left(K_{2 \sqrt{2} \delta}\right)^{4}}\left|f_{\mathbb{R}}\right|\left\|\delta_{h} \gamma_{\delta}\left(U^{\delta, n-1}\right)-\delta_{h} \gamma_{\delta}\left(u^{n-1}\right)\right\|_{0, h} \\
& \leq C_{\delta}\left(\left\|e_{U, \star}^{\delta, n-1}\right\|_{0, h}+\left|\delta_{h} u^{n-1}\right|_{\infty}\left\|e_{U}^{\delta, n-1}\right\|_{0, h}\right) \\
& \leq C_{\delta}\left[\left\|e_{U, \star}^{\delta, n-1}\right\|_{0, h}+\left(\tau^{2}+h^{2}\right)\right] \text {, }
\end{aligned}
$$

and

$$
\begin{aligned}
\left\|M^{n, 1}\right\|_{0, h} \leq & \left|\gamma_{\delta}\left(U^{\delta, n-1}\right)\right|_{\infty}\left\|\delta_{h} f\left(\gamma_{\delta}\left(U^{\delta, n-1}\right), \gamma_{\delta}\left(W^{\delta, n-1}\right)\right)-\delta_{h} f\left(\gamma_{\delta}\left(u^{n-1}\right), \gamma_{\delta}\left(w^{n-1}\right)\right)\right\|_{0, h} \\
\leq & C_{\delta}\left[\left\|\delta_{h} \gamma_{\delta}\left(U^{\delta, n-1}\right)-\delta_{h} \gamma_{\delta}\left(u^{n-1}\right)\right\|_{0, h}+\left\|\delta_{h} \gamma_{\delta}\left(W^{\delta, n-1}\right)-\delta_{h} \gamma_{\delta}\left(w^{n-1}\right)\right\|_{0, h}\right. \\
& \left.+\left(\left|\delta_{h} u^{n-1}\right|_{\infty}+\left|\delta_{h} w^{n-1}\right|_{\infty}\right)\left(\left\|e_{U}^{\delta, n-1}\right\|_{0, h}+\left\|e_{W}^{\delta, n-1}\right\|_{0, h}\right)\right] \\
\leq & C_{\delta}\left[\left\|e_{U, \star}^{\delta, n-1}\right\|_{0, h}+\left\|e_{W, \star}^{\delta, n-1}\right\|_{0, h}+\left(\tau^{2}+h^{2}\right)\right] .
\end{aligned}
$$


In addition, since $\max _{1 \leq n \leq N}\left|\delta_{h} f\left(u^{n-1}, w^{n-1}\right)\right|_{\infty}=\mathcal{O}(1)$, using Lemma 3.5 and the error estimate (3.30) we have

$$
\begin{aligned}
\left\|M^{n, 2}\right\|_{0, h} & \leq\left|\delta_{h} f\left(u^{n-1}, w^{n-1}\right)\right|_{\infty}\left\|\gamma_{\delta}\left(U^{\delta, n-1}\right)-\gamma_{\delta}\left(u^{n-1}\right)\right\|_{0, h} \\
& \leq C\left\|\gamma_{\delta}\left(U^{\delta, n-1}\right)-\gamma_{\delta}\left(u^{n-1}\right)\right\|_{0, h} \\
& \leq C_{\delta}\left\|e_{U}^{\delta, n-1}\right\|_{0, h} \\
& \leq C_{\delta}\left(\tau^{2}+h^{2}\right) .
\end{aligned}
$$

Thus, by (3.59)-(3.62), we arrive at

$$
\max _{2 \leq n \leq N}\left\|\delta_{h} \mu^{\delta, n}\right\|_{0, h} \leq C_{\delta}\left[\left\|e_{U, \star}^{\delta, n-1}\right\|_{0, h}+\left\|e_{W, \star}^{\delta, n-1}\right\|_{0, h}+\left(\tau^{2}+h^{2}\right)\right] .
$$

Proceeding in a similar way we, also, get

$$
\max _{2 \leq n \leq N}\left\|\delta_{h} \xi^{\delta, n}\right\|_{0, h} \leq C_{\delta}\left[\left\|e_{U, \star}^{\delta, n-1}\right\|_{0, h}+\left\|e_{W, \star}^{\delta, n-1}\right\|_{0, h}+\left(\tau^{2}+h^{2}\right)\right] .
$$

Thus, combining (3.57), (3.64) and (3.65), we get

$$
\nu_{\star}^{\delta, n} \leq\left(1+C_{\delta} \tau\right) \nu_{\star}^{\delta, n-1}+C \tau\left(\tau^{2}+h^{2}\right), \quad n=2, \ldots, N .
$$

Then, a standard discrete Grönwall argument along with (3.56) yield

$$
\max _{1 \leq n \leq N} \nu_{\star}^{\delta, n} \leq C_{\delta}\left(\tau^{2}+h^{2}\right) .
$$

The estimate (3.55) follows combining (3.66), (3.58) and (3.56).

We close this section showing an optimal order maximum norm error estimate for the approximations of the finite difference method.

Theorem 3.13. Let $\delta_{\star}$ be the constant defined by (3.16), $\delta_{0}>2 \delta_{\star},\left\{\left(U^{m}, W^{m}\right)\right\}_{m=0}^{N}$ be the approximations of the finite difference method (3.7)-(3.9), $\left\{\left(u^{m}, w^{m}\right)\right\}_{m=0}^{N}$ be defined by (3.1), $C_{S}$ be the constant in the inequality (3.4) and $C_{1, \delta_{0}}$ be the constant in the inequality (3.55) for $\delta=\delta_{0}$. If $\tau$ and $h$ are small enough such that

$$
\left(\tau^{2}+h^{2}\right) \sqrt{C_{S} C_{0, \delta_{0}}} \sqrt{C_{0, \delta_{0}}+C_{1, \delta_{0}}} \leq \delta_{\star},
$$

then there exist a constant $C>0$ which is independent of $\tau$ and $h$, and such that

$$
\max _{0 \leq n \leq N}\left(\left|U^{n}-u^{n}\right|_{\infty}+\left|W^{n}-w^{n}\right|_{\infty}\right) \leq C\left(\tau^{2}+h^{2}\right) .
$$

Proof. Let $\left\{\left(U^{\delta_{0}, m}, W^{\delta_{0}, m}\right)\right\}_{m=0}^{N}$ be the approximations of the modified finite difference method (3.20)(3.22) for $\delta=\delta_{0}$. The discrete Sobolev-type inequality (3.4) and the error estimates (3.30) and (3.55), yield that

$$
\max _{0 \leq n \leq N} \max \left\{\left|U^{\delta_{0}, n}-u^{n}\right|_{\infty},\left|W^{\delta_{0}, n}-w^{n}\right|_{\infty}\right\} \leq \sqrt{C_{S} C_{0, \delta_{0}}\left(C_{0, \delta_{0}}+C_{1, \delta_{0}}\right)}\left(\tau^{2}+h^{2}\right) .
$$

Now, from (3.69) and (3.67), we conclude that

$$
\begin{aligned}
\max _{0 \leq n \leq N} \max \left\{\left|U^{\delta_{0}, n}\right|_{\infty},\left|W^{\delta_{0}, n}\right|_{\infty}\right\} & \leq \delta_{\star}+\max _{0 \leq n \leq N} \max \left\{\left|u^{n}\right|_{\infty},\left|w^{n}\right|_{\infty}\right\} \\
& \leq 2 \delta_{\star} \\
& \leq \delta_{0} .
\end{aligned}
$$


Thus, according to Lemma 3.3, (3.70) yields $U^{\delta_{0}, n}=U^{n}$ and $W^{\delta_{0}, n}=W^{n}$ for $n=0, \ldots, N$, and the error estimate (3.68) is established by (3.69).

Remark 3.14. In the proof of Theorem 3.13, we use condition (3.67) along with the error estimates (3.30) and (3.55) to secure that $U^{\delta_{0}, n}=U^{n}$ and $W^{\delta_{0}, n}=W^{n}$ for $n=0, \ldots, N$ and a given $\delta_{0}>2 \delta_{\star}$. Thus, the error estimates (3.54) and (3.55) with $\delta=\delta_{0}$ establish an optimal order error estimate in the discrete $\mathrm{H}^{1}(0, L)$-norm $\|\cdot\|_{1, h}$, i.e.

$$
\max _{0 \leq n \leq N}\left(\left\|U^{n}-u^{n}\right\|_{1, h}+\left\|W^{n}-w^{n}\right\|_{1, h}\right) \leq C\left(\tau^{2}+h^{2}\right) .
$$

\section{NumericAL EXPERIMENTS}

\subsection{On the implementation of the finite difference method}

Let $n \in\{2, \ldots, N\}$. Then, we have

$$
\begin{aligned}
W^{n} & =-\frac{\tau}{1-i \alpha_{2} \tau} \delta_{h} U^{n}+\Psi^{n}, \\
U^{n} & =-\frac{\tau}{1-i \alpha_{1} \tau} \delta_{h} W^{n}+\Phi^{n},
\end{aligned}
$$

where

$$
\begin{aligned}
\Psi^{n} & :=\frac{1}{1-i \alpha_{2} \tau}\left[-\tau \delta_{h} U^{n-2}+\left(1+i \alpha_{2} \tau\right) W^{n-2}+i \lambda_{2} 2 \tau f\left(U^{n-1}, W^{n-1}\right) \otimes W^{n-1}\right], \\
\Phi^{n} & :=\frac{1}{1-i \alpha_{1} \tau}\left[-\tau \delta_{h} W^{n-2}+\left(1+i \alpha_{1} \tau\right) U^{n-2}+i \lambda_{1} 2 \tau f\left(U^{n-1}, W^{n-1}\right) \otimes U^{n-1}\right] .
\end{aligned}
$$

Now, combining (4.1a) and (4.1b), we obtain

$$
U^{n}-\lambda_{\star} \delta_{h}^{2} U^{n}=F^{n}
$$

where $\lambda_{\star}:=\frac{\tau^{2}}{\left(1-i \alpha_{1} \tau\right)\left(1-i \alpha_{2} \tau\right)}$ and $F^{n}:=-\frac{\tau}{1-i \alpha_{1} \tau} \delta_{h} \Psi^{n}+\Phi^{n}$. Thus, we can compute $W^{n}$ after computing $U^{n}$ avoiding the numerical solution of a linear system of algebraic equations. Observing that $\delta_{h}^{2} v_{j}=\frac{v_{j-2}-2 v_{j}+v_{j+2}}{4 h^{2}}$ for $j=1, \ldots, J+1$ and $v \in X_{h}$, it is easily seen that (4.2) is equivalent to the following linear system:

$$
D z-\frac{\lambda_{\star}}{4 h^{2}}\left[z_{J} \mathrm{e}^{J+1,1}+z_{J+1} \mathrm{e}^{J+1,2}+z_{1} \mathrm{e}^{J+1, J}+z_{2} \mathrm{e}^{J+1, J+1}\right]=b^{n}
$$

where: $z, b \in \mathbb{C}^{J+1}$ with $z_{j}=U_{j}^{n}$ and $b_{j}^{n}=F_{j}^{n}$ for $j=1, \ldots, J+1, D \in \mathbb{C}^{(J+1) \times(J+1)}$ is a 5 -diagonal invertible matrix, and, for $i \in \mathcal{I}:=\{1,2, J, J+1\}$, $\mathrm{e}^{J+1, i} \in \mathbb{R}^{J+1}$ with $\left(\mathrm{e}^{J+1, i}\right)_{j}=\delta_{i j}$ for $j=1, \ldots, J+1$. Since $D$ is invertible, (4.3) is equivalent to

$$
z-\frac{\lambda_{\star}}{4 h^{2}}\left[z_{J} D^{-1} \mathrm{e}^{J+1,1}+z_{J+1} D^{-1} \mathrm{e}^{J+1,2}+z_{1} D^{-1} \mathrm{e}^{J+1, J}+z_{2} D^{-1} \mathrm{e}^{J+1, J+1}\right]=D^{-1} b^{n} .
$$

Computing, first, the vectors $D^{-1} b^{n}$ and $\left(D^{-1} \mathrm{e}^{J+1, i}\right)_{i \in \mathcal{I}}$, we are able to compute $z_{1}, z_{2}, z_{J}$ and $z_{J+1}$ by solving a $4 \times 4$ system of algebraic equations consisting of the first two and the last two rows of (4.4). In the sequel, we can compute the unknowns $\left(z_{j}\right)_{j=3}^{J-1}$ directly from (4.4). We note that, since the matrix $D$ is independent of $n$, only the vector $D^{-1} b^{n}$ has to be computed at each time step. However, the first upper and the first low diagonal of matrix $D$ has only zero elements. To use that sparsity of $D$ to decrease the memory needed and the complexity of computing the finite difference approximations, we consider the case that $J+1$ is even, i.e., 
$J=2 M-1$ for some $M \in \mathbb{N}$. Then, we define $z^{e} \in \mathbb{C}^{M}$ with $z_{j}^{e}=z_{2 j}$ for $j=1, \ldots, M$, and $z^{o} \in \mathbb{C}^{M}$ with $z_{j}^{o}=z_{2 j-1}$ for $j=1, \ldots, M$. It is easily seen that (4.3) is equivalent to the following $M \times M$ linear systems:

$$
\widetilde{D} z^{e}-\frac{\lambda_{\star}}{4 h^{2}}\left[z_{M}^{e} \mathrm{e}^{M, 1}+z_{1}^{e} \mathrm{e}^{M, M}\right]=b^{n, e} \quad \text { and } \quad \widetilde{D} z^{o}-\frac{\lambda_{\star}}{4 h^{2}}\left[z_{M}^{o} \mathrm{e}^{M, 1}+z_{1}^{o} \mathrm{e}^{M, M}\right]=b^{n, o}
$$

where: $\widetilde{D} \in \mathbb{C}^{M \times M}$ is a 3-diagonal invertible matrix, $b^{n, e}, b^{n, o} \in \mathbb{C}^{M}$ with $b_{j}^{n, e}=F_{2 j}^{n}$ and $b_{j}^{n, o}=F_{2 j-1}^{n}$ for $j=1, \ldots, M$, and, $\mathrm{e}^{M, 1}, \mathrm{e}^{M, M} \in \mathbb{C}^{M}$ with $\left(\mathrm{e}^{M, i}\right)_{j}=\delta_{i j}$ for $j=1, \ldots, M$ and $i \in\{1, M\}$. Using the invertibility of $\widetilde{D}$, we write (4.5) equivalently as

$$
\begin{aligned}
& z^{e}-\frac{\lambda_{\star}}{4 h^{2}}\left[z_{M}^{e} \widetilde{D}^{-1} \mathrm{e}^{M, 1}+z_{1}^{e} \widetilde{D}^{-1} \mathrm{e}^{M, M}\right]=\widetilde{D}^{-1} b^{n, e}, \\
& z^{o}-\frac{\lambda_{\star}}{4 h^{2}}\left[z_{M}^{o} \widetilde{D}^{-1} \mathrm{e}^{M, 1}+z_{1}^{o} \widetilde{D}^{-1} \mathrm{e}^{M, M}\right]=\widetilde{D}^{-1} b^{n, o} .
\end{aligned}
$$

First we compute the vectors $\widetilde{D}^{-1} b^{n, e}, \widetilde{D}^{-1} b^{n, o}, \widetilde{D}^{-1} \mathrm{e}^{M, 1}$ and $\widetilde{D}^{-1} \mathrm{e}^{M, M}$. Then, we compute $z_{1}^{e}$ and $z_{M}^{e}$ solving a $2 \times 2$ system of algebraic equations consisting of the first and last row of system (4.6a). Also, we compute $z_{1}^{o}$ and $z_{M}^{o}$ solving a $2 \times 2$ system of algebraic equations consisting of the first and last row of system (4.6b). Finally, we compute the unknowns $\left(z_{j}^{e}\right)_{j=2}^{M-2}$ directly from (4.6a) and the unknowns $\left(z_{j}^{o}\right)_{j=2}^{M-2}$ directly from (4.6b). We note that, since the matrix $\widetilde{D}$ is independent of $n$, only the vectors $\widetilde{D}^{-1} b^{n, e}$ and $\widetilde{D}^{-1} b^{n, o}$ have to be computed at each time step.

The case $n=1$ is similar and thus is omitted.

\subsection{Numerical results}

We wrote a program, called DRC3FD, that computes the finite difference approximations implementing the algorithm that is based on the numerical solution of the linear systems (4.6) requiring $J+1$ to be an even integer. The program uses the programming language FORTRAN 77 and double precision arithmetic; also it calls the LINPACK subroutine zgtsl to solve 3-diagonal linear systems and the LINPACK subroutines zgefa and zgesl to solve general $2 \times 2$ linear systems. When the exact solution is known, first we choose $J+1=2 N$ and then we compute the global discrete $\mathrm{L}^{\infty}$ error $\mathcal{E}_{\infty}(N):=\max _{0 \leq n \leq N} \max \left\{\left|U^{n}-u^{n}\right|_{\infty},\left|W^{n}-w^{n}\right|_{\infty}\right\}$ and the global discrete $\mathrm{L}^{2}$ error $\mathcal{E}_{0}(N):=\max _{0 \leq n \leq N} \max \left\{\left\|U^{n}-u^{n}\right\|_{0, h},\left\|W^{n}-w^{n}\right\|_{0, h}\right\}$. The experimental rate of convergence for two successive values $N_{1}$ and $N_{2}$ of $N$ with corresponding errors $\mathcal{E}\left(N_{1}\right)$ and $\mathcal{E}\left(N_{2}\right)$, is then computed by the formula: $\log \left(\frac{\mathcal{E}\left(N_{1}\right)}{\mathcal{E}\left(N_{2}\right)}\right) / \log \left(\frac{N_{2}}{N_{1}}\right)$.

\subsubsection{Example 1}

For a parameter $\Lambda \in(-1,1)$, we define functions $A, B: \mathbb{R} \rightarrow \mathbb{R}$ by

$$
A(x):=\mu_{A} \frac{\cosh (\mu x)}{1+\Lambda \cosh (2 \mu x)} \quad \text { and } \quad B(x):=\mu_{B} \frac{\sinh (\mu x)}{1+\Lambda \cosh (2 \mu x)}
$$

for $x \in \mathbb{R}$, where $\mu:=\sqrt{1-\Lambda^{2}}, \mu_{A}:=\sqrt{2(1+\Lambda)} \mu$ and $\mu_{B}:=\sqrt{2(1-\Lambda)} \mu$. It is well known (see, e.g., [2,3]), that the functions

$$
u_{\star}(x, t)=\mathrm{e}^{-\mathrm{i} \Lambda t} A(x) \quad \text { and } \quad w_{\star}(x, t)=i \mathrm{e}^{-\mathrm{i} \Lambda t} B(x)
$$

form a solution pair of the Dirac system (1.1) when $f(u, w)=|u|^{2}-|w|^{2}, \lambda_{1}=-\lambda_{2}=1$ and $\alpha_{2}=-\alpha_{1}=1$. Observing that $\left|u_{\star}(x, 0)\right|+\left|w_{\star}(x, 0)\right|=O\left(10^{-15}\right)$ for $|x| \geq 50$, we can consider that $u_{\star}$ and $w_{\star}$ have compact support in the space interval $[-50,50]$. Thus, we test the efficiency of our method, by computing the finite difference approximations choosing $L=100, u(x, 0)=u_{\star}(x+50,0)$ and $w(x, 0)=w_{\star}(x+50,0)$ for $x \in[0,100]$, 
TABLE 1. Example 1: rates of convergence in the discrete $\mathrm{L}^{\infty}$ and $\mathrm{L}^{2}$ norms.

\begin{tabular}{|c|c|c|c|c|}
\hline$N$ & $\mathcal{E}_{\infty}(N)$ & Rate & $\mathcal{E}_{0}(N)$ & Rate \\
\hline 64 & $4.103744(-1)$ & - & $5.066743(-1)$ & - \\
128 & $4.407355(-2)$ & 3.219 & $8.386832(-2)$ & 2.595 \\
256 & $1.028941(-2)$ & 2.099 & $1.991953(-2)$ & 2.074 \\
512 & $2.535220(-3)$ & 2.021 & $4.923096(-3)$ & 2.016 \\
1024 & $6.327513(-4)$ & 2.031 & $1.229362(-4)$ & 2.030 \\
\hline
\end{tabular}

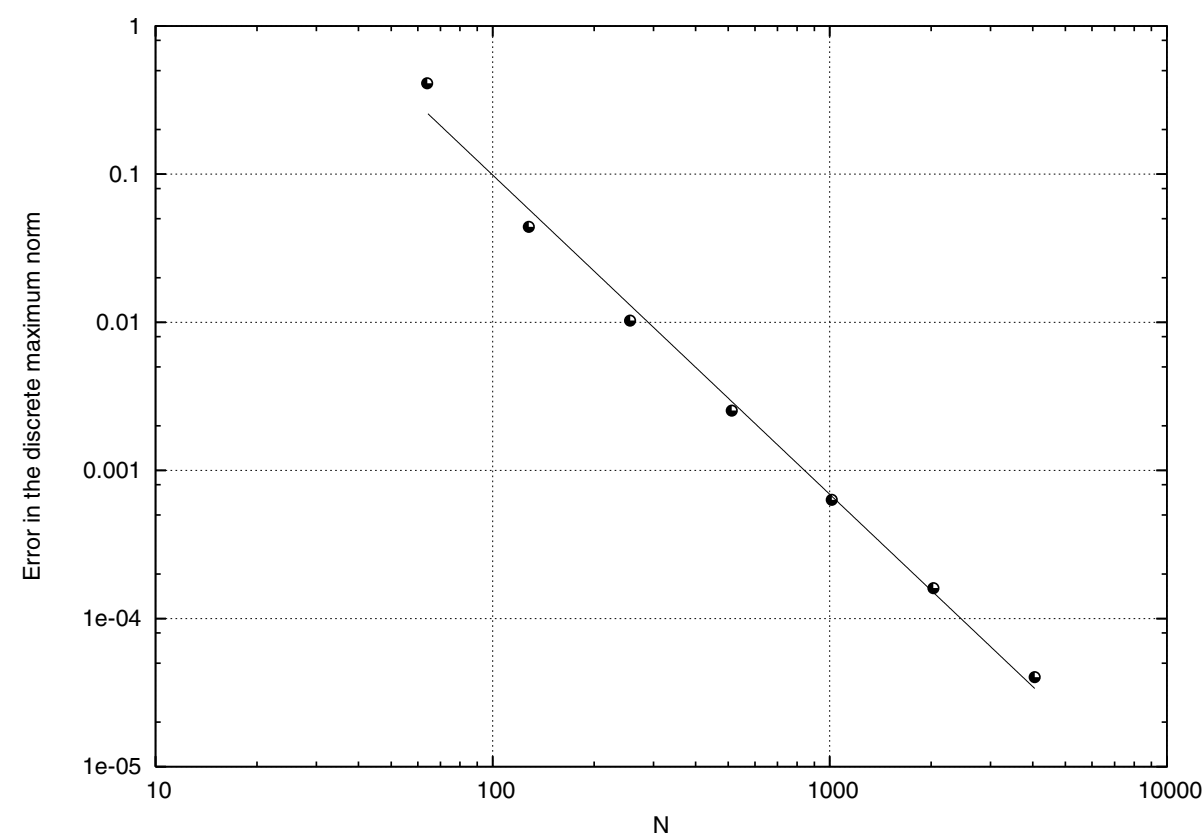

Figure 1. Example 1: $\log (N) / \log \left(\mathcal{E}_{\infty}(N)\right)$ plot of errors along with their linear least square fitting (slope $=-2.115)$.

and then by comparing them with the nodal values of the functions $u(x, t)=u_{\star}(x+50, t)$ and $w(x, t)=$ $w_{\star}(x+50, t)$. In the numerical experiments, we set $T=8.0, \Lambda=\frac{3}{4}$ and $N=\frac{J+1}{2}=64,128,256,512,1024$. The results obtained are displayed in Table 1 and Figure 1, and confirm a second order experimental order of convergence in the discrete $\mathrm{L}^{\infty}$ and $\mathrm{L}^{2}$ norms. In Figures 2 and 3 we display the exact solution to the problem along with its finite difference approximation at the final time $T$.

\subsubsection{Example 2}

Let $u_{\star}$ and $w_{\star}$ be the functions defined by (4.7). Also, let $v \in(-1,1)$ and $\gamma:=\frac{1}{\sqrt{1-v^{2}}}$. It is well known (see, e.g., $[2,27])$ that the following functions

$$
\begin{aligned}
& u_{S}(x, t)=\left(\frac{\gamma+1}{2}\right)^{\frac{1}{2}} u_{\star}(\gamma(x-v t), \gamma(t-v x))+\operatorname{sign}(v)\left(\frac{\gamma-1}{2}\right)^{\frac{1}{2}} w_{\star}(\gamma(x-v t), \gamma(t-v x)), \\
& w_{S}(x, t)=\left(\frac{\gamma+1}{2}\right)^{\frac{1}{2}} w_{\star}(\gamma(x-v t), \gamma(t-v x))+\operatorname{sign}(v)\left(\frac{\gamma-1}{2}\right)^{\frac{1}{2}} u_{\star}(\gamma(x-v t), \gamma(t-v x))
\end{aligned}
$$



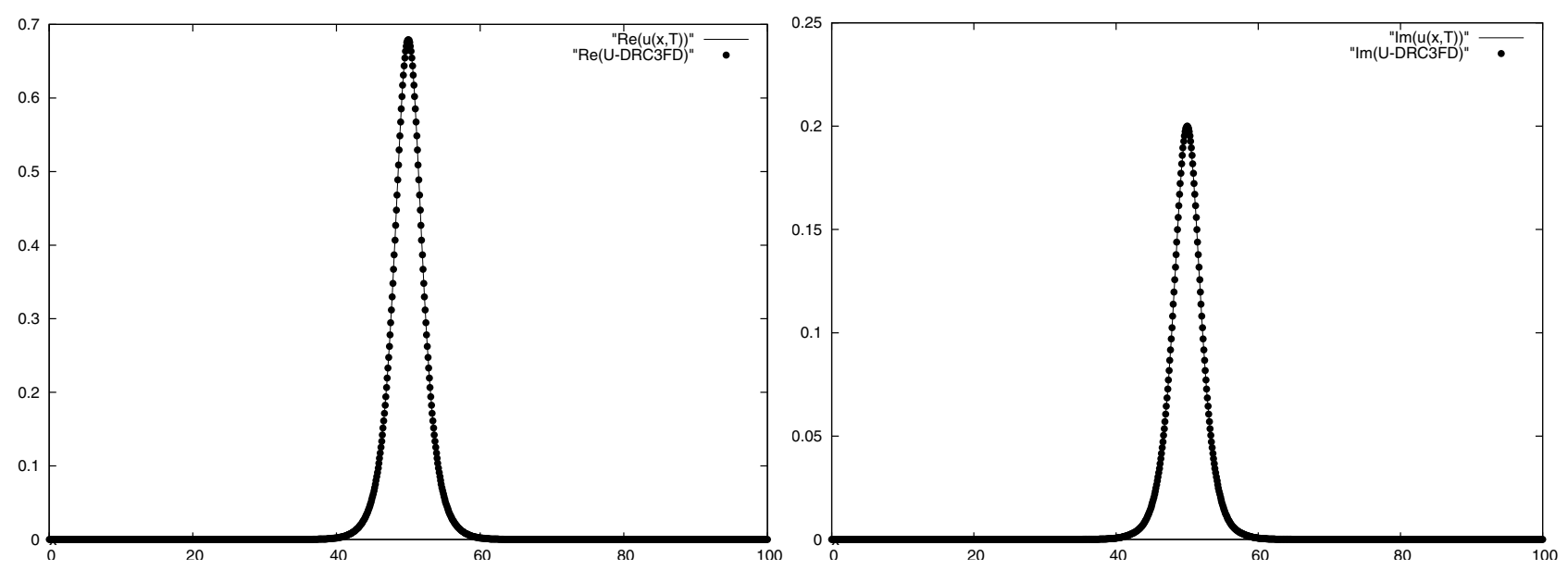

Figure 2. Example 1: $u(\cdot, T)$ and $U^{N}$ with $T=8.0$ and $N=\frac{J+1}{2}=512$.
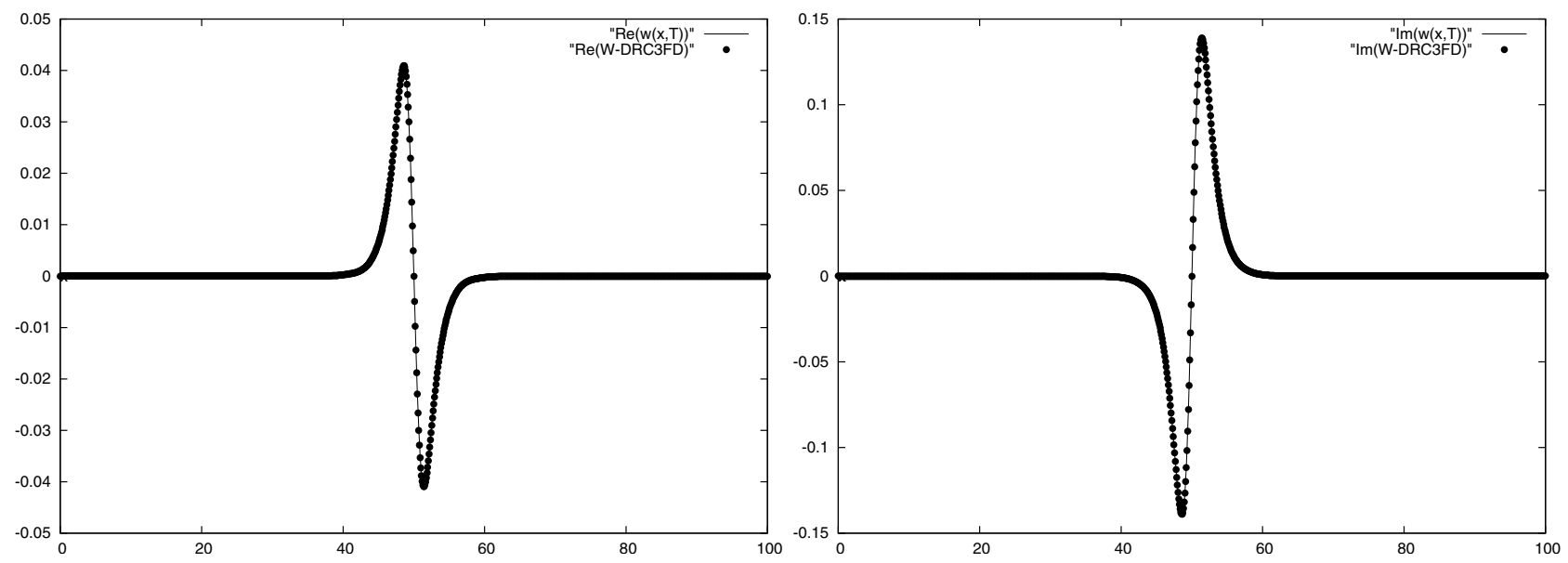

Figure 3. Example 1: $w(\cdot, T)$ and $W^{N}$ with $T=8.0$ and $N=\frac{J+1}{2}=512$.

form another solution pair of the Dirac system (1.1) when $f(u, w)=|u|^{2}-|w|^{2}, \lambda_{1}=-\lambda_{2}=1$ and $\alpha_{2}=-\alpha_{1}=1$. Here, $\operatorname{sign}(v):=\frac{v}{|v|}$ when $v \neq 0$, and $\operatorname{sign}(v):=0$ for $v=0$. We test our method, by computing the finite difference approximations choosing $L=40, u(x, 0)=u_{S}(x+20,0)$ and $w(x, 0)=w_{S}(x+20,0)$ for $x \in[0,40]$, and then by comparing them with the nodal values of the functions $u(x, t)=u_{S}(x+20, t)$ and $w(x, t)=w_{S}(x+20, t)$. In the numerical experiments, we set $T=8.0, \Lambda=\frac{3}{4}, v=\frac{1}{2}$ and $N=\frac{J+1}{2}=64,128,256,512,1024,2028$. The results obtained are displayed in Table 2 and Figure 4, and confirm a second order experimental order of convergence in the discrete $\mathrm{L}^{\infty}$ and $\mathrm{L}^{2}$ norms. Finally, Figures 5 and 6 display the exact solution to the problem along with its finite difference approximation at the final time $T$. 
TABLE 2. Rates of convergence in the discrete $L^{\infty}$ and $L^{2}$ norms for Example 2.

\begin{tabular}{|c|c|c|c|c|}
\hline$N$ & $\mathcal{E}_{\infty}(N)$ & Rate & $\mathcal{E}_{0}(N)$ & Rate \\
\hline 64 & $1.351699(-1)$ & - & $2.377012(-1)$ & - \\
128 & $2.971434(-2)$ & 2.185 & $5.842444(-2)$ & 2.024 \\
256 & $7.302886(-3)$ & 2.025 & $1.449370(-2)$ & 2.011 \\
512 & $1.821913(-3)$ & 2.003 & $3.616602(-3)$ & 2.003 \\
1024 & $4.582936(-4)$ & 1.991 & $9.090737(-4)$ & 1.992 \\
2028 & $1.161667(-4)$ & 2.008 & $2.305187(-4)$ & 2.008 \\
\hline
\end{tabular}

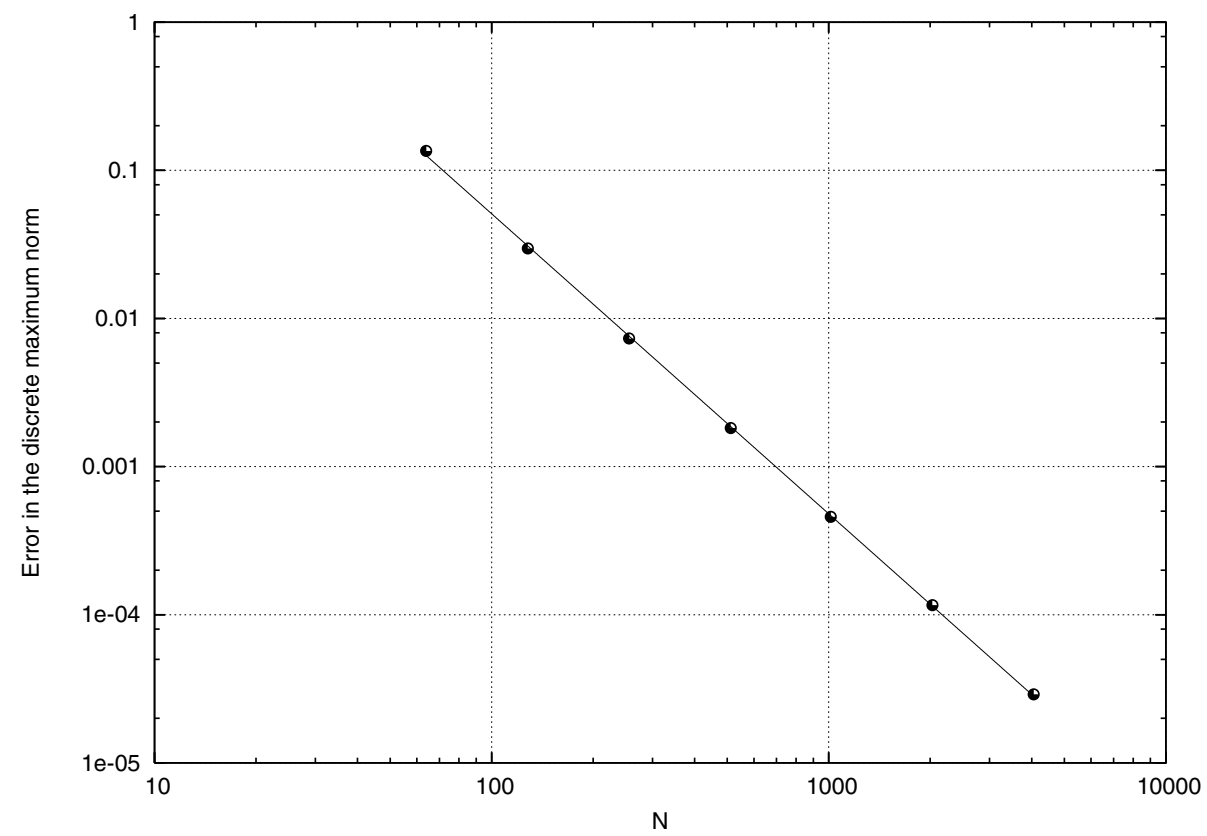

Figure 4. Example 2: $\log (N) / \log \left(\mathcal{E}_{\infty}(N)\right)$ plot of errors along with their linear least square fitting (slope $=-2.025$ ).
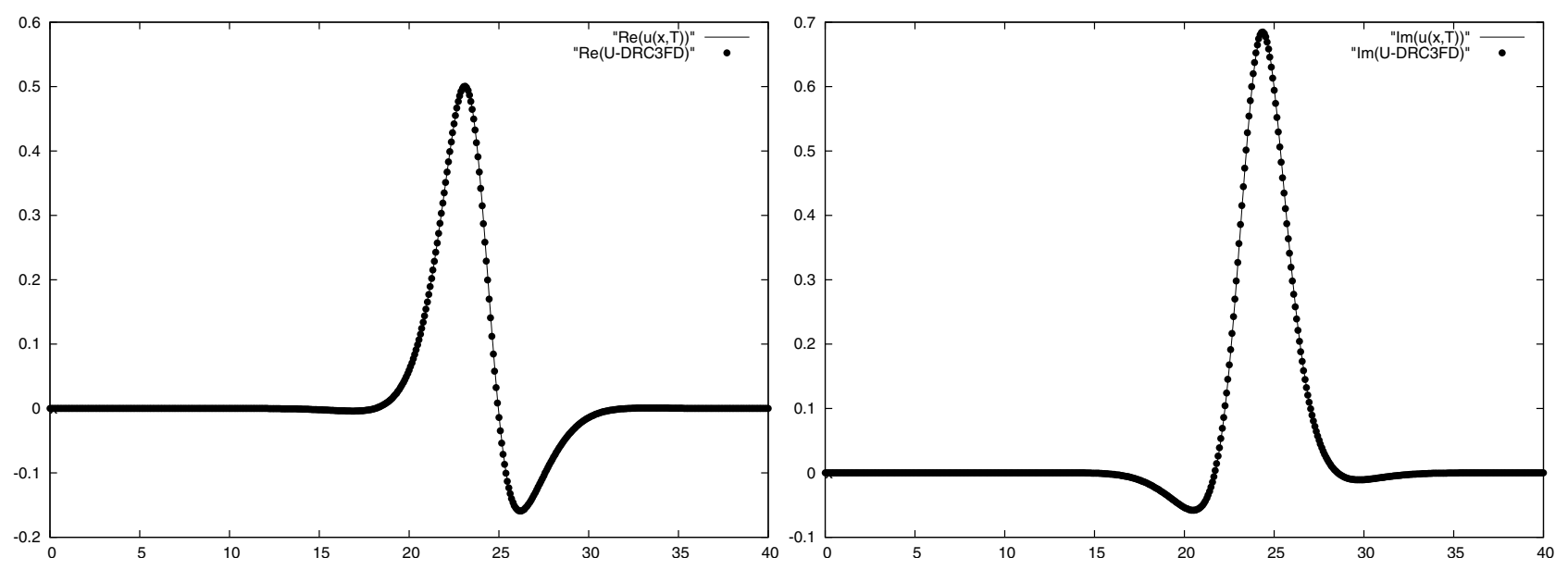

FiguRE 5. Example 2: $u(\cdot, T)$ and $U^{N}$ with $T=8.0$ and $N=\frac{J+1}{2}=256$. 

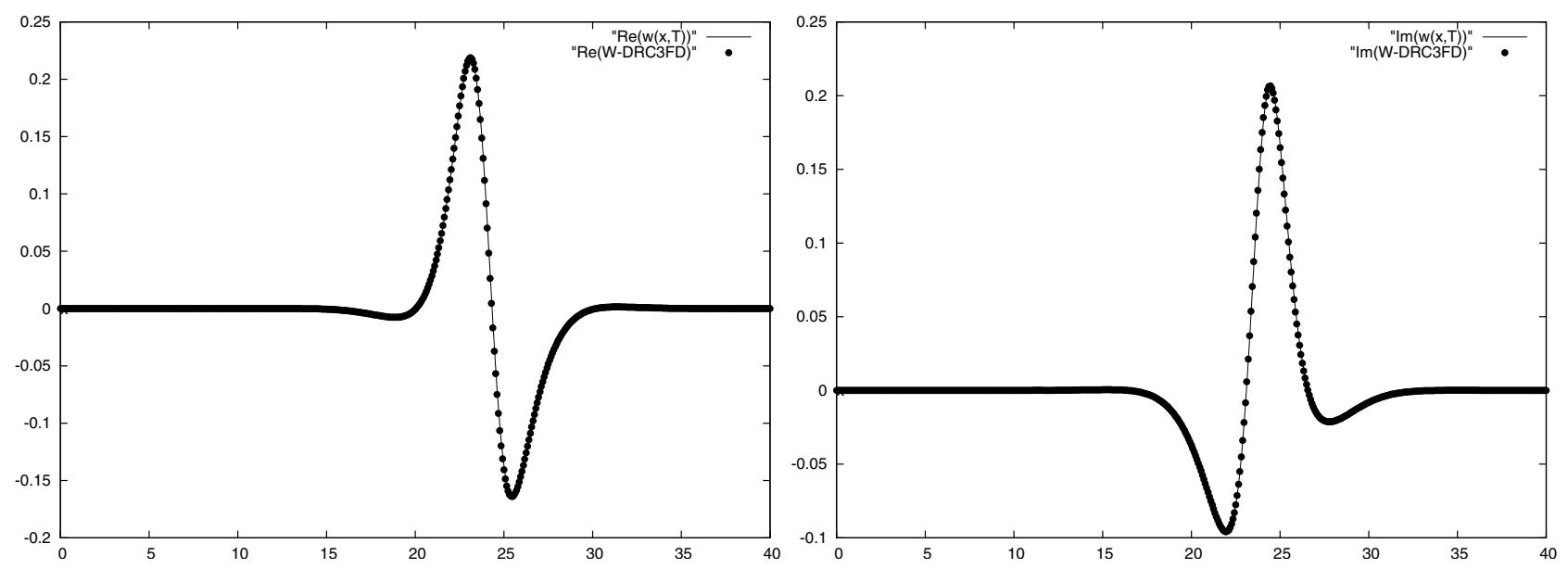

Figure 6. Example 2: $w(\cdot, T)$ and $W^{N}$ with $T=8.0$ and $N=\frac{J+1}{2}=256$.

Acknowledgements. The authors would like to thank the referees for their helpful comments and suggestions. The second author has been partially supported by the FP7-REGPOT-2009-1 project 'Archimedes Center for Modeling, Analysis and Computation'.

\section{REFERENCES}

[1] A. Alvarez, Linearized Crank-Nicholson scheme for nonlinear Dirac equations. J. Comput. Phys. 99 (1992) 348-350.

[2] A. Alvarez and B. Carreras, Interaction dynamics for the solitary waves of a nonlinear Dirac model. Phys. Lett. A 86 (1981) $327-332$.

[3] A. Alvarez, Pen-Yu Kuo and L. Vazquez, The numerical study of a nonlinear one-dimensional Dirac equation. Appl. Math. Comput. 13 (1983) 1-15.

[4] N. Bournaveas, Local and global solutions for a nonlinear Dirac system. Advances Differential Equations 9 (2004) 677-698.

[5] N. Bournaveas, Local well-posedness for a nonlinear Dirac equation in spaces of almost critical dimension. Discrete Contin. Dyn. Syst. Ser. A 20 (2008) 605-616.

[6] N. Boussaid, P. D'Ancona and L. Fanelli, Virial identity and weak dispersion for the magnetic Dirac equation. J. Math. Pures Appl. 95 (2011) 137-150.

[7] J. De Frutos, Estabilidad y convergencia de esquemas numericos para sistemas de Dirac no lineales. Revista Internacional de Métodos Numéricos para Cálculo y Diseño en Ingenieria 5 (1989) 185-202.

[8] J. De Frutos and J.M. Sanz-Serna, Split-step spectral schemes for nonlinear Dirac systems. J. Comput. Phys. 83 (1989) 407-423.

[9] V. Delgado, Global solutions of the Cauchy problem for the classical Coupled Maxwell-Dirac and other nonlinear Dirac equations in one space dimension. Proc. Amer. Math. Soc. 69 (1978) 289-296.

[10] T. Dupont, Galerkin methods for first order hyperbolics: an example. SIAM J Numer. Anal. 10 (1973) 890-899.

[11] R.T. Glassey, On one-dimensional coupled Dirac equations. Trans. Amer. Math. Soc. 231 (1977) 531-539.

[12] B.-Y. Guo, J. Shen and C.-L. Xu, Spectral and pseudospectral approximations using Hermite functions: application to the Dirac equation. Adv. Comput. Math. 19 (2003) 35-55.

[13] J. Hong and C. Li, Multi-symplectic Runge-Kutta methods for nonlinear Dirac equations. J. Comput. Phys. 211 (2006) $448-472$.

[14] L. Hörmander, Lectures on Nonlinear Hyperbolic Differential Equations. Springer-Verlag (1997).

[15] S. Jiménez, Derivation of the discrete conservation laws for a family of finite difference schemes. Appl. Math. Comput. 64 (1994) 13-45.

[16] T. Kato, Nonlinear semigroups and evolution equations. J. Math. Soc. Japan 19 (1967) 508-520.

[17] S. Machihara, One dimensional Dirac equation with quadratic nonlinearities. Discrete Contin. Dyn. Syst. Ser. A 13 (2005) 277-290.

[18] S. Machihara, Dirac equation with certain quadratic nonlinearities in one space dimension. Commun. Contemp. Math. 9 (2007) 421-435.

[19] S. Machihara, M. Nakamura and T. Ozawa, Small global solutions for nonlinear Dirac equations. Differential Integral Equations 17 (2004) 623-636. 
[20] S. Machihara, M. Nakamura, K. Nakanishi and T. Ozawa, Endpoint Strichartz estimates and global solutions for the nonlinear Dirac equation. J. Funct. Anal. 219 (2005) 1-20.

[21] S. Machihara, K. Nakanishi and K. Tsugawa, Well-posedness for nonlinear Dirac equations in one dimension. Kyoto J. Math. 50 (2010) 403-451.

[22] A. Majda, Compressible fluid flow and systems of conservation laws in several space variables. Appl. Math. Sci. 53 (1984).

[23] E. Salusti and A. Tesei, On a semi-group approach to quantum field equations. Nuovo Cimento A 2 (1971) 122-138.

[24] I. E. Segal, Non-linear semi-groups. Ann. of Math. 78 (1963) 339-364.

[25] S. Shao and H. Tang, Higher-order accurate Runge-Kutta discontinuous Galerkin methods for a nonlinear Dirac model. Discrete Contin. Dyn. Syst. Ser. B 6 (2006) 623-640.

[26] B. Thaller, The Dirac equation, Texts and Monographs in Physics. Springer-Verlag, Berlin, Heidelberg (2010).

[27] H. Wang and H. Tang, An efficient adaptive mesh redistribution method for a non-linear Dirac equation. J. Comput. Phys. 222 (2007) 176-193.

[28] G.E. Zouraris, On the convergence of a linear conservative two-step finite element method for the nonlinear Schrödinger equation. ESAIM: M2AN 35 (2001) 389-405. 